\title{
13C Metabolic Flux Analysis at a Genome-Scale
}

\author{
Saratram Gopalakrishnan ${ }^{1}$ and Costas D. Maranas ${ }^{1 *}$ \\ ${ }^{1}$ Department of Chemical Engineering, Pennsylvania State University, University Park, PA, USA \\ * Corresponding Author: Costas D. Maranas \\ Phone: (814) 863-9958 \\ Fax: (814) 865-7846 \\ E-mail: costas@psu.edu
}




\section{ABSTRACT}

Metabolic models used in 13C metabolic flux analysis generally include a limited number of reactions primarily from central metabolism. They typically omit degradation pathways, complete cofactor balances, and atom transition contributions for reactions outside central metabolism. This study addresses the impact on prediction fidelity of scaling-up mapping models to a genome-scale. The core mapping model employed in this study accounts for (75 reactions and 65 metabolites) primarily from central metabolism. The genome-scale metabolic mapping model (GSMM) (697 reaction and 595 metabolites) is constructed using as a basis the iAF1260 model upon eliminating reactions guaranteed not to carry flux based on growth and fermentation data for a minimal glucose growth medium. Labeling data for 17 amino acid fragments obtained from cells fed with glucose labeled at the second carbon was used to obtain fluxes and ranges. Metabolic fluxes and confidence intervals are estimated, for both core and genome-scale mapping models, by minimizing the sum of square of differences between predicted and experimentally measured labeling patterns using the EMU decomposition algorithm.

Overall, we find that both the topology and estimated values of the metabolic fluxes remain largely consistent between the core and GSMM. Stepping up to a genome-scale mapping model leads to wider flux inference ranges for 20 key reactions present in the core model. The glycolysis flux range doubles due to the possibility of active gluconeogenesis, the TCA flux range expanded by $80 \%$ due to the availability of a bypass through arginine consistent with labeling data, and the transhydrogenase reaction flux was essentially unresolved due to the presence of as many as five routes for the inter-conversion of NADPH to NADH afforded by the genome-scale model. By globally accounting for ATP demands in the GSMM model the unused ATP decreased drastically with the lower bound matching the maintenance ATP requirement. A non-zero flux for the arginine degradation pathway was identified to meet biomass precursor demands as detailed in the iAF1260 model. Inferred ranges for $81 \%$ of the reactions in the genome-scale metabolic (GSM) model varied less than one-tenth of the basis glucose uptake rate (95\% confidence test). This is because as many as 411 reactions in the GSM are growth coupled meaning that the single measurement of biomass formation rate locks the reaction flux values. 
This implies that accurate biomass formation rate and composition are critical for resolving metabolic fluxes away from central metabolism and suggests the importance of biomass composition (re)assessment under different genetic and environmental backgrounds. In addition, the loss of information associated with mapping fluxes from MFA on a core model to a GSM model is quantified.

Keywords: Metabolic Flux Analysis; Metabolic network; EMU algorithm; Genome-scale; E. coli

\section{INTRODUCTION}

Cellular metabolism is a direct indicator of its physiological state (Nielsen, 2003). Estimation of fluxes using $13 \mathrm{C}$ metabolic flux analysis involves solving a nonlinear least-squares problem for the flux distribution capable of matching experimentally measured labeling patterns of analyzed metabolites (Zomorrodi et al., 2012), typically amino-acids and fatty acids. Labeling patterns given a flux distribution can be predicted by relating the target labeling patterns to input tracers and a flux distribution using a system of algebraic equations. This can be achieved by decomposing the network using various frameworks such as isotopomers (Schmidt et al., 1997), cumomers (Wiechert et al., 1999), or the EMU method (Antoniewicz et al., 2007a) all of which are based on the atom mapping matrix (AMM) concept (Zupke and Stephanopoulos, 1994). The computational complexity arises from the fact that the number of equations scales super-linearly with network size. Network decomposition using the isotopomer approach results in 4,612 unknown mass isotopomers involving bilinear terms for a complete central metabolic network of E. coli (Antoniewicz et al., 2007a). Efforts in the last decade have focused on reducing complexity and proposing better algorithms to solve the mass isotopomer distributions (MIDs) (Wiechert and de Graaf, 1996) (Wiechert et al., 1997). For instance, the EMU method reduces the number of isotopomer variables from 4,612 to 310 for a central metabolic network of E.coli. Sub-networks can be simplified further using the Dulmage-Mendelsohn decomposition to improve the speed of estimation (Young et al., 2008). A variety of optimization approaches (Schmidt et al., 1999) have been used to infer the metabolic fluxes that minimize the sum of the least squares while the statistical significance of the estimated flux distribution is evaluated using

the $\chi^{2}$ test(Pazman, 1993). Due to the limited number of analyzed metabolites and inherent measurement error, flux ranges rather than unique values are obtained for the metabolic fluxes using either linearized statistics (Mollney et al., 1999), grid search, or non-linear statistics 
(Antoniewicz et al., 2006). All of these approaches are iterative in nature, requiring repeated solution of the least-squares minimization problem placing additional computational burden.

The general practice is for 13-C MFA models to include only a skeletal representation of central metabolism comprised of the EMP pathway, PPP, TCA cycle, glyoxylate shunt, and the ED pathway. Important pathways such as serine and arginine degradation are typically absent. 13C MFA has been used extensively to elucidate the metabolic properties of knockout strains (Flores et al., 2002; Hua et al., 2003; Shimizu, 2004; Usui et al., 2012; Zhao and Shimizu, 2003), identify metabolic bottlenecks (Antoniewicz et al., 2007b), and even confirm the activity of various pathways (Crown et al., 2011; You et al., 2014; Young et al., 2011). Earlier E.coli mapping models (Shimizu, 2004; Zhao and Shimizu, 2003) did not use a biomass equation, instead they included specific drains proportional to the specific growth rate (Hua et al., 2003) to account for biomass formation (Holms, 1996). Newer models use a defined biomass equation obtained from macromolecular composition of a wild-type strain (Antoniewicz et al., 2007b). While the use of a biomass equation in MFA constrains metabolism by imposing specific requirements on macromolecule precursors from central metabolism, it neglects the contribution of the soluble pool and the energetic requirements that are part of a genome-scale model. The use of cofactor balances in MFA, limited to newer prokaryotic models (Bonarius et al., 1998; van Gulik and Heijnen, 1995), can sharpen the reaction bounds involved in energy metabolism. However, such MFA models assume that the cell is purely biosynthetic neglecting possibly active pathways such as gluconeogenesis and amino acid degradation. MFA models for organisms such as synechocystis (Young et al., 2011), CHO cells, (Ahn and Antoniewicz, 2011), and hybridoma cell lines (Murphy et al., 2013) have so far omitted cofactor balances. Nevertheless, it has been previously shown that neglecting potentially active reactions that contribute to cofactor balances can alter the estimated flux ranges using 13C MFA (Bonarius et al., 1998). The key advantage of using a genome-scale model in MFA is that it represents the totality of reactions that can be carried out by the organism avoiding any biases introduced by lumping reactions or omitting pathways pre-judged as non-functional. An earlier MFA study on a larger metabolic model of E.coli (Suthers et al., 2007) proposed the possibility of the integration of a number of non-central pathways, and found that nearly half of the fluxes were fixed by stoichiometry alone. Here we take the next step by making use of a genome-scale model 
for flux elucidation thereby avoiding any pre-conceived assumptions about which pathways should be active or inactive.

Mapping models used for MFA typically include less than $10 \%$ of the reactions contained within a genome-scale model. Flux ranges obtained using 13C MFA have been used extensively to test the validity of genome-scale models (Chen et al., 2011; Dash et al., 2014; Saha et al., 2012). However, this transfers the assumptions used in the construction of MFA models to the GSM model, thereby providing a solution space which may be more constrained than what the labeling data supports. On the other hand, GSMs are generally analyzed using methods such as Flux Balance Analysis (Varma and Palsson, 1994), Flux Variability Analysis (Mahadevan and Schilling, 2003), and MOMA (Segre et al., 2002). Often, the predicted metabolic phenotypic space is quite large with split ratios and cycles poorly resolved. 13C MFA at a genome-scale holds the promise of resolving split ratios and cycles while avoiding making any assumptions about which pathways should be active. As a result, it can identify the activity of all degradation pathways which are generally neglected by existing mapping models, impose detailed cofactor balances, generate unbiased confidence intervals for all fluxes within the network, provide insight into which fluxes can or cannot be resolved using C-13 labeling data (i.e., identifiability problem), maintain consistency with a comprehensive biomass equation describing metabolite demands for macromolecule biosynthesis, soluble pool, and experimentally measured energy demands, and even accurately predict the impact of genetic modifications which are essentially unresolvable by constraint-based modeling techniques (Copeland et al., 2012).

Successful 13C-MFA at a genome-scale requires a reliable GSM model along with detailed atom maps of every reaction in the network. Atom mapping information for central metabolism reactions is readily available from biochemistry textbooks. For other pathways, online databases such as KEGG (Latendresse et al., 2012), MetaCyc (Korner and Apostolakis, 2008), or MetRxn (Kumar et al., 2012) are useful resources. MetRxn includes reaction mapping information for over 27,000 reactions generated using a novel sub-structure search algorithm known as Canonical Labeling for Clique Approximation (CLCA) (Kumar and Maranas, 2014) which offers improved accuracy and memory utilization over existing heuristic algorithms. The approach utilizes number theory to generate unique ids for each atom followed by a maximum common substructure search. The MetRxn database contains atom mapping information for reactions from 
112 metabolic models including iAF1260 directly downloadable from http://www.metrxn.che.psu.edu/.

In this study we carry out estimation of flux ranges for E-coli using both a core mapping model (Leighty and Antoniewicz, 2013) and for a genome-scale model iAF1260 (Feist et al., 2007) using measured fluxes and 13C labeling data as constraints. The GSM model is refined further by imposing measured extracellular fluxes as constraints and then performing flux variability analysis (FVA) to identify the part of the network that can carry non-zero fluxes. Subsequently, active sub-networks of different size are generated by decomposing the network using the EMU algorithm (Antoniewicz et al., 2007a). The fluxes are estimated by solving a least squares problem involving the minimization of the sum of squares of difference between predicted metabolite labeling and experimentally observed metabolite labeling patterns. $95 \%$ confidence intervals are generated by varying the fluxes individually until the minimum sum of squares exceeds a pre-defined threshold. We demonstrate how a combination of the constraints involved in FBA and 13C-MFA can be used in a concerted manner to effectively resolve fluxes through key branch points such as the oxidative pentose phosphate pathway, the Entner-Doudoroff pathway, and the glyoxylate shunt. Our results allude to the possibility of coexistence of anabolic and catabolic reactions and bypasses resulting in expanded ranges for many reactions which were previously reported to be precisely inferred. They also shed light on the inability of MFA alone to resolve alternate pathways and energy metabolism when the entirety of metabolic reactions implied by the GSM model is used. Surprisingly, we found that results are largely insensitive to biomass composition fluctuations as the experimental error in the labeling data is the dominant source of prediction uncertainty. The impact of using a core model for MFA is quantitatively assessed by contrasting the corresponding flux ranges. In addition, the loss of information when fluxes derived from MFA in the core metabolic model are directly ported on a GSM model is assessed and discussed.

\section{METHODS}

\subsection{Genome-scale atom mapping model}

The genome-scale metabolic model of E. coli (Feist et al., 2007) consisting of 2,382 reactions and 1,670 metabolites was pruned by eliminating reactions incapable of carrying flux for the bioprocess data measured by Leighty et.al. (Leighty and Antoniewicz, 2013). The model was 
further simplified by manually eliminating thermodynamically infeasible cycles disjoint from the metabolic network. The resultant model has 697 reactions (29 reversible reactions) and 595 metabolites with glucose as the sole carbon source. Examples of reactions eliminated from the model include uptake systems of other carbon sources, beta-oxidation, and nucleotide salvage pathways in agreement with Suthers, et al (Suthers et al., 2007). Atom mapping information for the core model was obtained from the study conducted by Leighty, et al (Leighty and Antoniewicz, 2013). Atom mapping information for the genome-scale model was obtained using the CLCA algorithm (Kumar and Maranas, 2014).

\subsection{Flux estimation procedure}

Network decomposition was accomplished using the EMU algorithm that relates the labeling pattern of the input tracer and a flux distribution to a labeling pattern of all analyzed intracellular metabolites. Fluxes were estimated by solving a non-linear least squares problem described in detail in Appendix A. This problem minimizes the variance-weighted sum of the squares of differences between the predicted and experimentally observed labeling patterns for 18 fragments from 10 different intracellular amino acids subject to flux non-negativity. Glucose labeled at the second carbon with $99.5 \%$ purity was used as the tracer input in the analyzed dataset (Leighty and Antoniewicz, 2013) as it was found to best resolve oxidative PPP. All other carbon atoms were assumed to contain the heavy isotope of carbon equal to the natural abundance. The least squares objective function $\boldsymbol{\phi}$ (see Appendix A) depends on the subset of fluxes $(\boldsymbol{w})$ present in the EMU model which is a subset of all fluxes $(\boldsymbol{v})$ in the S-matrix of the metabolic model. Since the system of component balance equations describing the metabolic network is underdetermined, the set of fluxes $\boldsymbol{v}$ can be expressed in terms of the free fluxes $\boldsymbol{u}$ by means of a null-space decomposition (Antoniewicz et al., 2006). Consequently, the set of fluxes describing the EMU model $\boldsymbol{w}$ and the objective function $\boldsymbol{\phi}$ can also be expressed in terms of the subset of free fluxes $\boldsymbol{u}$. This allows for the estimation of all the fluxes within the metabolic network by the resolution of the free fluxes $\boldsymbol{u}$. The problem described in Appendix A was solved using the fmincon function from the optimization toolbox of MATLAB. A user-supplied Hessian matrix was provided for the interior point algorithm (Byrd et al., 2000; Byrd et al., 1999; Waltz et al., 2006) of fmincon using the procedure introduced by Antoniewicz, et al (Antoniewicz et al., 2006). Given the nonconvex nature of the objective function, the problem was solved 100 
times and the best solution was selected as the optimal flux distribution candidate. This solution was selected as the optimal flux distribution for further analysis only if it satisfied two criteria: the optimization problem converged to the same solution at least 70 times out of 100 runs and the obtained flux distribution was unaffected by local perturbations. All fluxes (mmol/dmol-glc) are reported using $100 \mathrm{mmol}$ of glucose uptake per gram dry cell weight as the basis. Fluxes were also estimated with the amino acid MS data obtained using glucose labeled at the fifth carbon as well to clearly identify loss of resolution due to model scale-up.

\subsection{Confidence intervals}

The underdetermined nature of the metabolic network could result in multiple flux distributions with the same labeling pattern. Furthermore, metabolite labeling measurements are inherently noisy introducing error in the data that further contributes to metabolic flux inference uncertainty. To this end, we estimated the lower and upper bounds of the $95 \%$ confidence interval of each flux such that the sum of squares of residuals (SSRES) is within 3.84 of the minimum SSRES. The value 3.84 corresponds to the $\chi^{2}$ statistic for a p-value of 0.05 and one degree of freedom. The lower and upper bounds were estimated using an iterative procedure (Antoniewicz et al., 2006) where every flux $\mathrm{v}_{\mathrm{j}}$ is successively varied up (or down for lower bound) and a new best flux distribution is re-calculated. The upper (or lower) bound for $\mathrm{v}_{\mathrm{j}}$ defining the $95 \%$ confidence interval corresponds to the value that renders the difference between the re-calculated and original SSRES's equal to 3.84. Since the genome-scale model contains a large number of measurement-coupled reactions, a flux coupling analysis (Burgard et al., 2004) is performed to identify the list of all reactions coupled to an extracellular measurement (i.e., 411 out of a total of 697 reactions). These reactions are assigned a range consistent with the extracellular measurement variance to which they are fully coupled. In addition, a flux coupling analysis between every reaction pair in the metabolic model further reduces the number of reactions whose range needs to be estimated based on the procedure described above. We identified 250 coupled reaction pairs in the EMU-balanced network implying that there were only 186 remaining reaction fluxes whose confidence levels needed to be directly assessed. Additional flux range reduction was achieved by successively performing FVA (Mahadevan and Schilling, 2003) using the obtained 95\% confidence level ranges as flux 
bounds. The technical details of the procedure describing the implementation for 13C-MFA for GSM models is described in Appendix A.

\section{RESULTS}

\subsection{Active EMU network}

Decomposition of the genome-scale network using the EMU algorithm resulted in EMU subnetworks of sizes 1 through 9 (i.e., number of carbons in the EMU fragment). The network consisted of 1,400 balanced EMUs and 3,526 EMU reactions, spanning 432 out of the 726 fluxes in the GSM model. Of the 3,526 EMU reactions, 1,405 reactions were duplicates, contributed by redundant mappings. In comparison, the core model consists of 310 balanced EMUs and 863 EMU reactions with 181 duplicates, spanning 80 out of the 100 fluxes in the model. It is interesting to note that a nine-fold scaling up of the mapping model resulted in only a five-fold increase in the number of EMUs, a four-fold increase in the number of EMU reactions, and a three-fold increase in the number of unique EMU reactions. This moderate increase is because only 256 out of 595 metabolites (present in 426 out of 726 fluxes) are required to predict the experimentally observed labeling patterns. $65 \%$ of all the fluxes involved in EMU balances are from central metabolism and amino acid metabolism. The remaining 35\% result from the contributions of cofactor biosynthesis, lipid biosynthesis, and nucleotide biosynthesis, accounting for novel carbon transformations absent in the core model. In comparison, the core EMU network includes all reactions from central metabolism and a limited number of reactions from amino acid metabolism including Serine hydroxymethyltransferase (SHMT), glycine cleavage system, and threonine aldolase. The GSMM model sheds light on novel carbon transformations, alternate atom mapping pathways and redundant atom maps, which provide the means to explain the experimentally measured labeling patterns better, and highlight the role of assumptions involved in flux estimation using the core model.

The novel carbon transformations allowed by the pathways outside central and amino acid metabolism in the GSM model stems from the production of molecules such as $\mathrm{CO}_{2}$, glycoaldehyde, and formate which are eventually recycled into central metabolism. $\mathrm{CO}_{2}$ is produced as a by-product of the synthesis of several cofactors and trace metabolites such as 
coenzyme A, thiamine pyrophosphate, heme, pyridoxal phosphate, menaquinol 8, and NAD. In addition, $\mathrm{CO}_{2}$ is also produced by the decarboxylation of serine to phosphatidyl ethanolamine. The core model only accounts for $\mathrm{CO}_{2}$ production and consumption within the central metabolic reactions. Similarly, formate is absent in the core model, whereas, it is produced by the degradation of formyl-tetrahydrofolate, and biosynthesis of tetrahydrofolate, riboflavin, and thiamine pyrophosphate in the GSM model. Glycolate, which is absent in the core model, is produced as a by-product of tetrahydrofolate biosynthesis in the GSM model. Since the FVA flux ranges for these reactions indicate a non-zero lower bound, it provides quantitative evidence that these often ignored transformations play a role in explaining the observed labeling data.

The GSMM model also traces alternate routes of existing pathways in the core model. For example, the production of succinate from $\alpha$-ketoglutarate occurs only through the TCA cycle in the core model, whereas the GSMM allows for two additional routes: the degradation of glutamate through the $\gamma$-aminobutyrate pathway and the degradation of arginine. The key difference between these three pathways is the energy output. The TCA cycle route produces one NADH and one ATP, whereas the $\gamma$-aminobutyrate pathway route produces only one NADH and the arginine pathway produces one NADH but requires one ATP to recycle the consumed acetylCoA. Since the atom transitions for succinate are identical for all three pathways, 13C-MFA will fail to resolve fluxes between these pathways thus the core model arbitrarily apportions the entire flux towards succinate through the TCA cycle. Resolution between the three parallel pathways can be achieved only after cofactor balances are included. A similar diversity of metabolic routes implied by the GSM model can be seen with the production of pyruvate from succinate. The core model only includes the pathway involving malic enzyme whereas the GSM model accounts for an additional route through propionyl-CoA with an identical energy output implying nonidentifiability between the two alternatives. Such alternate routes with identical atom mapping are ubiquitous in GSM models thereby resulting in non-identifiability or poor resolution of misleadingly well resolved fluxes according to the core model.

Multiple metabolic reactions are sometimes described by exactly the same EMU reaction leading to non-identifiability. We identified 195 such instances among the 726 reactions in the GSM model. The complete list of EMU reactions consisted of 122 duplicate reactions, 85 triplicate reactions, 27 quadruplicate reactions, and 48 EMU reactions describing identically five or more 
reactions from the genome-scale model. The source of this redundancy can be traced back to four factors: (i) isozymes with different cofactors, (ii) alternate reactions facilitating the same atom transfer, and (iii) group transfer reactions such as transaminases. For example, isozymes of Malic Enzyme catalyze the oxidation of malate to pyruvate using either NAD or NADP as a cofactor. Thus, the EMU reaction describing the atom transfer reaction from malate to pyruvate is identical for both isozymes. The elongation of the fatty acid chain releases one $\mathrm{CO}_{2}$ from malonyl-ACP in the first step of the cycle. As a result, the corresponding EMU reaction occurs eleven times accounting for all the fatty-acid chain elongation reactions. The conversion of glutamate to $\alpha$-ketoglutarate occurs through the transaminase reactions for which glutamate is the amino group donor. The GSM model contains 18 different aminotransferase reactions thereby resulting in multiple EMU reactions producing $\alpha$-ketoglutarate from glutamate. Similarly, the EMU reaction describing the carbon transfer from ATP to ADP (or AMP) arises in an identical manner for up to 94 reactions in the GSM model. Therefore, all GSM reactions that map to the same EMU reaction cannot be resolved by MFA alone as only the sum of their respective flux values is constrained.

\subsection{Flux identifiability and statistical validity of the model}

Because the EMU network spans a much smaller fraction of the GSM model, the number of $\chi^{2}$ degrees of freedom (DOF) for the regression model differs significantly depending on whether it is defined with respect to the entire GSM model or with respect to the EMU network alone. Table 1 shows the comparison of the $\chi^{2}$ degrees of freedom for the core model and the GSM model. The number of DOF is defined as the difference between the number of data points (measured fluxes and metabolite mass fractions) and the number of free variables in the network (EMU and GSM networks). Statistical significance of inference requires that the estimated minimum sum of squares of residuals (SSRES) be within an expected range determined by the confidence level and the number of DOF for the model.

This gives rise to 55 DOF for the core model. The core model contains 20 reactions (20\% of the all fluxes within the metabolic network) that do not provide EMU balances of which 15 are fully coupled to an extracellular flux measurement. Since the number of variables outside the EMU balances included in the least-squares regression model is very small, the impact on the expected sum of squares of residuals (SSRES) is minimal, thereby ensuring that it is safe to use the entire 
metabolic network as the regression model. With a non-negative DOF, the core model seldom encounters issues with statistical validity of the estimated fluxes.

In contrast, the DOF for GSM model is -30 after a cursory analysis. This is because the number of reactions unaccounted by EMU balances for the GSM is considerably larger causing the number of free fluxes in the entire metabolic network to exceed the number of available data points. A negative DOF for the GSM model would imply over-fitting and lack of statistical significance. However, the EMU network only spans about $60 \%$ of the metabolic network.

Table 1: $\chi^{2}$ degrees of freedom for the core model and the genome-scale model. Statistical significance requires that the number of degrees of freedom be positive as a $\chi^{2}$ value is defined only for positive integers. The difference in the number of degrees of freedom estimated using free fluxes and based on the EMU network points to an inherent flaw in using the free fluxes as the number of model parameters.

\begin{tabular}{|l|ll|}
\hline & Degrees of Freedom & Maximum SSRES \\
\hline Core model & 55 & 96 \\
$\begin{array}{l}\text { Genome-scale model } \\
\text { (based on free fluxes) }\end{array}$ & -30 & Not defined \\
$\begin{array}{l}\text { Genome-scale model } \\
\text { (based on EMU network) }\end{array}$ & 27 & 44 \\
\hline
\end{tabular}

Analyzing the EMU network associated with the GSM model revealed that the regression model has 27 DOF. This non-negative value of the DOF arise due to a $40 \%$ reduction in the number of variables (i.e., free fluxes) and a large increase in the number of fluxes coupled to an extracellular flux measurement. It was found that 256 out of the 595 balanced metabolites were involved in EMU balances. Of these, 214 metabolites were completely balanced by reactions involved in EMU balances, meaning that they did not feed into non-EMU pathways. Of the 42 metabolites feeding into other pathways, 28 of them were consumed by measurement-coupled pathways only, indicating that only 14 metabolites required additional equality constraints to be considered balanced EMU metabolites. Therefore, the actual EMU model contains 439 reactions 
and 242 balanced metabolites. Further reduction of the model by elimination of measurementcoupled reactions revealed that there are only 99 free fluxes. In comparison, the entire metabolic network has 274 free fluxes. Therefore, 13C-MFA can be performed on this model if there are at least 99 data points. Since the data set used in this analysis contains 126 data points, the leastsquares fitting approach can be safely applied to obtain statistically acceptable fits. Also shown in Table 1 is the maximum allowed SSRES for an accepted fit with 95\% confidence. An increase in the number of variables causes a reduction in the degrees of freedom, as a result of which the maximum allowed SSRES for 95\% confidence is reduced for the GSM model.

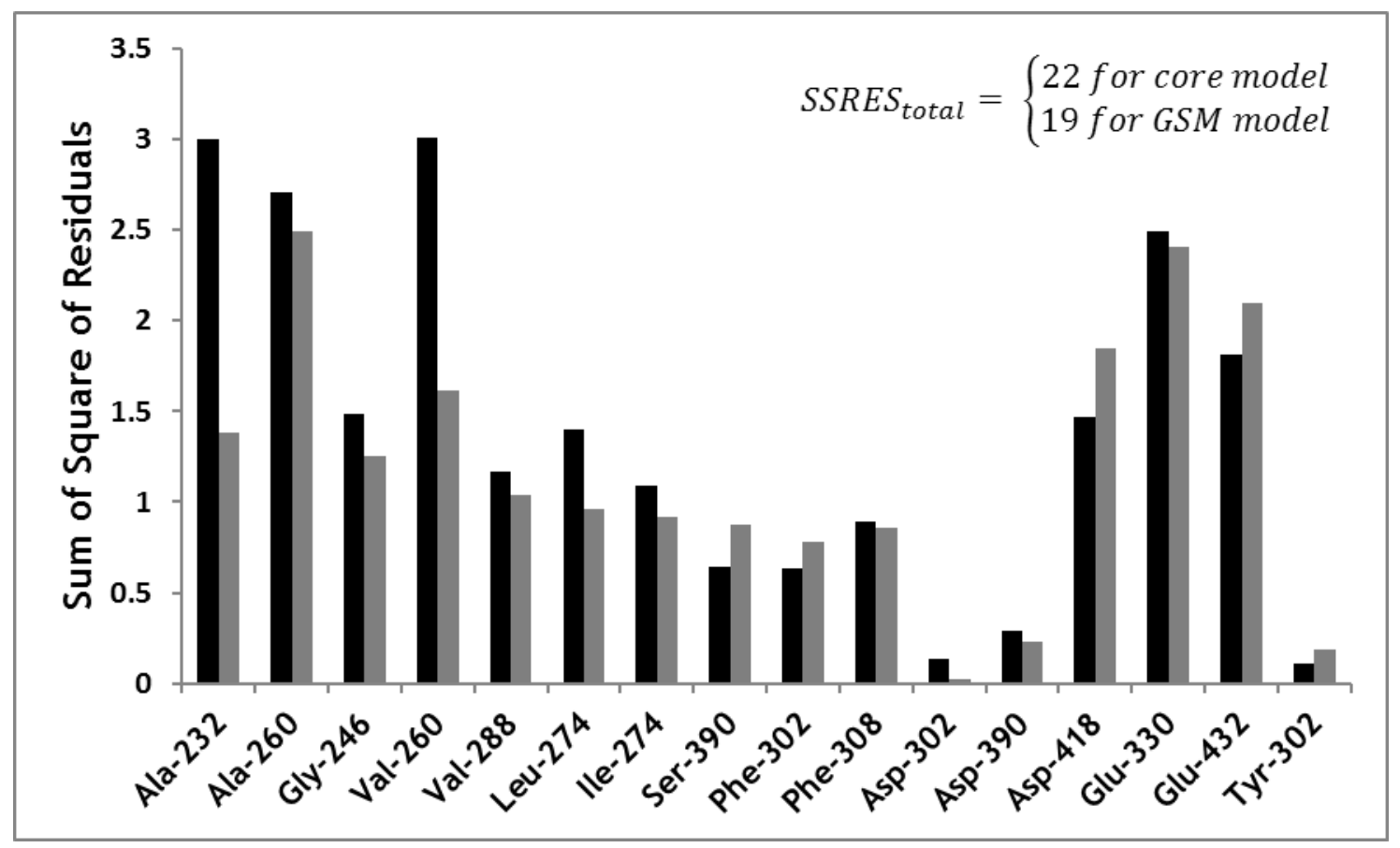

Figure 1: Comparison of prediction of experimentally observed amino acid MS data by the core model (๑) and the GSM model (•).

\subsection{Flux and range estimation at the genome-scale}

Flux elucidation using the GSMM model predicts the experimentally observed MS data better than the core model (see Figure 1). This improved prediction is attributed to the improved prediction of alanine and valine MS measurements with minimal changes to the quality of prediction of other labeling patterns. Improvements for alanine and valine are due to changes in the estimated fluxes in central metabolism (see also Figure $2 b, c, d$ and e). The inclusion of alternate pathways, new carbon mapping information, and complete metabolite and cofactor 
balances in the GSMM model results in significant changes in the PPP and wider flux ranges for reactions in glycolysis and the TCA cycle. The ED pathway and glyoxylate shunt flux ranges remained similar in both the core model and GSM model. Identical trends were observed upon analysis with a glucose tracer labeled at the fifth carbon (Supplementary figure 1).

Among the glycolytic reactions (Figure 2a and b) flux through PGI was unaffected. However, the remaining reactions had expanded flux ranges due to the inclusion of gluconeogenesis and alternate pathways of pyruvate metabolism. An unaffected PGI flux range indicates that $13 \mathrm{C}$ MFA using a GSMM model is capable of resolving the glycolysis/PPP split ratio despite the large increase in the number of reactions. Expanded flux ranges for TPI, GAPD, PGM, and ENO arise from the presence of an alternate pathway from dihydroxyacetone phosphate to pyruvate through methylglyoxal. However, the alternate methylglyoxal pathway involves a different energy balance yielding no ATP and less NADH than the EMP pathway, thus limiting its upper bound to $20 \mathrm{mmol} / \mathrm{dmol}$-glc. As a consequence of this, the glycolytic reactions have a non-zero lower bound of $65 \mathrm{mmol} / \mathrm{dmol}$-glc. Both the lower and upper bounds of PYK are altered significantly. The lower bound of PYK drops to $5 \mathrm{mmol} / \mathrm{dmol}$-glc. Two factors contribute to this reduction: (i) the availability of the phosphotransferase system (PTS) for glucose uptake as an alternative to PYK, and (ii) a significant flux through the anaplerotic reaction PPC which serves to replenish TCA metabolites. The inferred non-zero lower bound for PYK suggests that the alternate pathways (methylglyoxal and PTS) can only carry a fraction of the flux in lower glycolysis. The upper bound of PYK increases to $141 \mathrm{mmol} / \mathrm{dmol}$-glc due to the presence of a futile cycle with PPS (carrying a maximum flux of $20 \mathrm{mmol} / \mathrm{dmol}-\mathrm{glc}$ ) resulting in the hydrolysis of one ATP per unit flux through this cycle. A similar effect was also observed with the phosphorylation of glucose where G6PP can carry at most $20 \mathrm{mmol} / \mathrm{dmol}$-glc of flux, thus increasing the upper bound of the PTS and HK reactions by the same amount. The impact of gluconeogenesis is manifested in the flux range of PFK. A decreased lower bound of this reaction compared to the core model is due to the reduced contribution of the non-oxidative PPP towards fructose-6-phosphate production. The increased upper bound of this reaction is due to the activity of the FBP reaction from gluconeogenesis. With the upper bound of the FBP reaction limited to $8 \%$ of the total glucose input to the network the upper bound of PFK is increased by the same amount to account for this fully resolved in the GSMM futile cycle. 
(a)

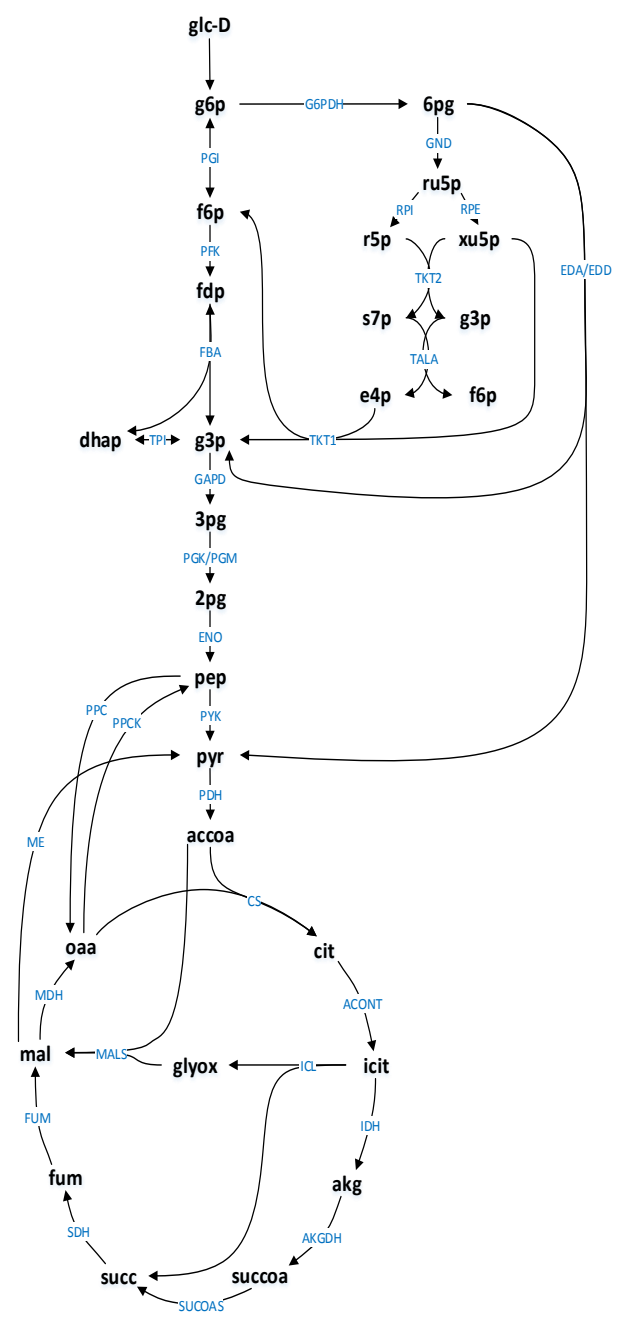

(b)

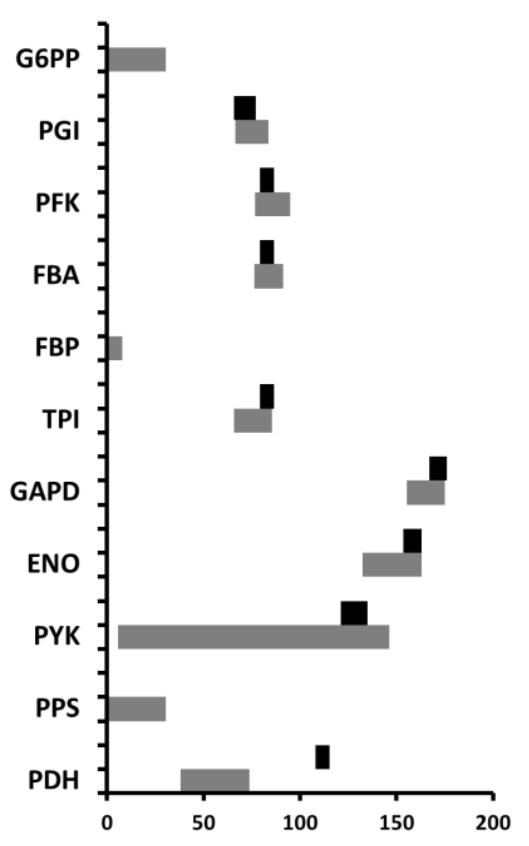

(c)

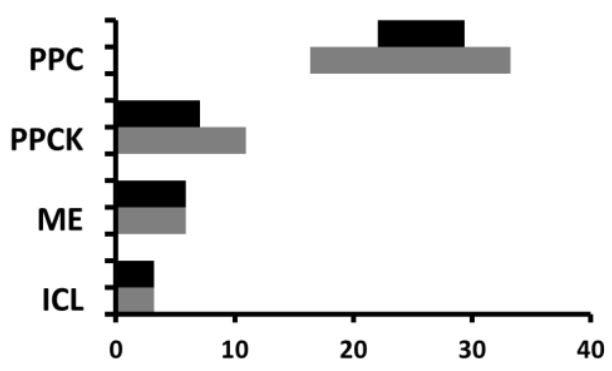

(d)

(e) 

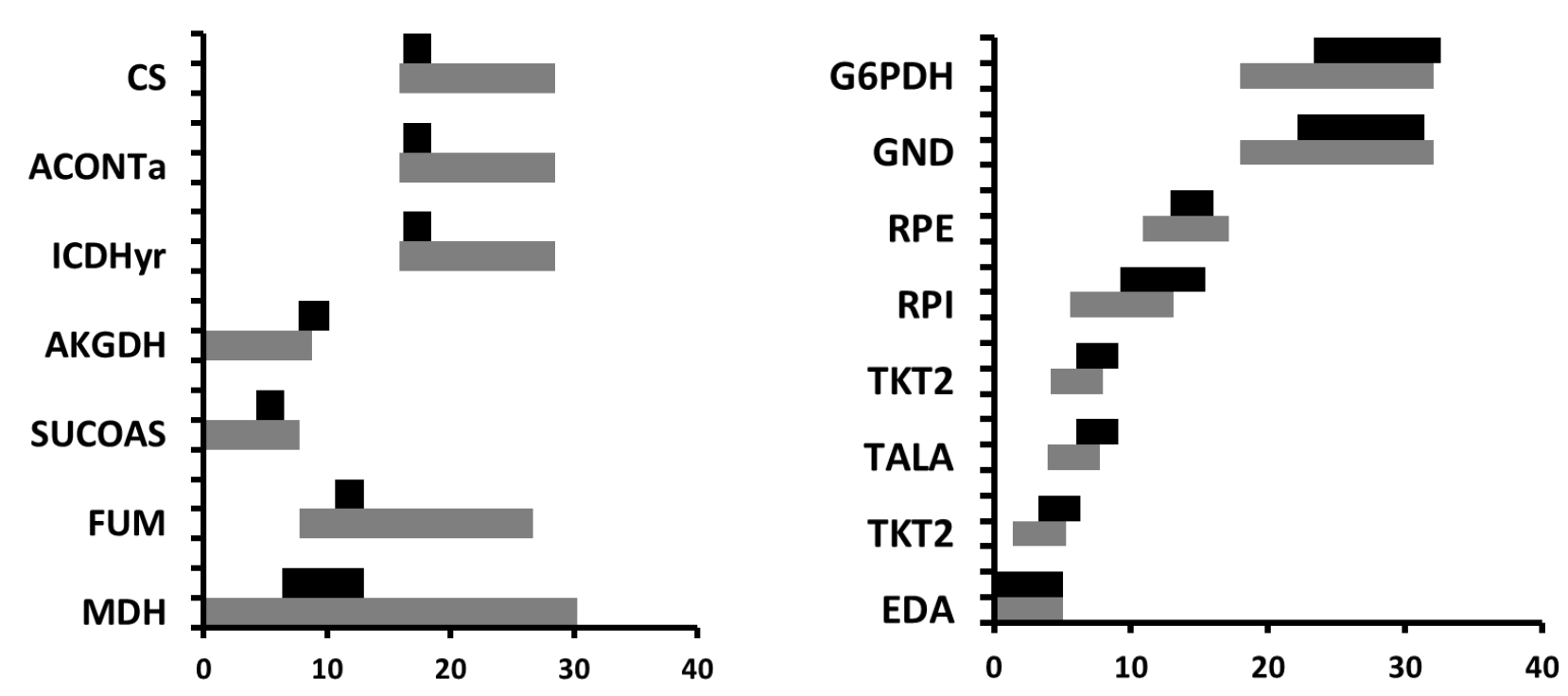

Figure 2: Flux distribution comparison for core model and GSM model. (a) Schematic representation of all reactions and metabolites involved in central metabolism of E. coli. Comparison of flux ranges (in mmol/dmol-glucose) using (-) core model and ( $\bullet$ ) GSM model for (b) glycolysis and gluconeogenesis, (c) anaplerotic reactions and glyoxylate shunt, (d) TCA cycle, (e) PPP and ED pathway.

Among the monophosphate shunts (PPP and ED pathways; Figure 2a and e), G6PDH and GND reactions showed a small difference between the core model and GSMM models to account for the increased glucose-6-phosphate demand for glycogen synthesis in the genome-scale model. In contrast, a significant shift was observed in the non-oxidative PPP reactions: TKT1, TKT2 and TALA. Both the lower and upper bounds of these reactions were reduced by a factor of 2.5 mmol/dmol-glc, implying reduced carbon flux through this pathway. This is a consequence of increased drains for biomass components R5P, S7P, and E4P in accordance with the biomass composition in the GSM model. Specifically, S7P was diverted towards lipopolysaccharide biosynthesis, R5P was shunted towards tetrahydrofolate biosynthesis and nucleotides, and E4P was used in pyridoxal phosphate and aromatic amino acids synthesis. Under the experimental stated growth condition, these drains amounted to $1 \%, 0.2 \%$, and $0.04 \%$ of the total glucose uptake for S7P, R5P, and E4P, respectively. In addition, some Ru5P was diverted for lipopolysaccharide biosynthesis amounting to a drain of $1 \%$ of the total glucose uptake. In contrast, the core model only contains drains for R5P for nucleotide biosynthesis and E4P for 
aromatic amino acid biosynthesis thereby predicting higher fluxes through the non-oxidative branch of the PPP. These differences arise from the fact that the biomass equation for the core model neglects the soluble pool and other cell wall components, which constitute up to $12 \%$ of the cell dry weight (Long and Antoniewicz, 2014).

Loss of flux identifiability when scaling up to the GSMM was manifested in the TCA cycle and associated fluxes (Figure $2 \mathrm{a}$ and $\mathrm{d}$ ). This inability to resolve fluxes was primarily due to the presence of various alternate pathways between metabolites. Flux through PDH was lower due to a required flux through pyruvate oxidase (POX) which converts pyruvate directly to acetate. In fact, because Acetyl-CoA can be fully produced using the ACK reaction this allows for a complete bypass of PDH. The lower bound of AKGDH decreased to zero due to the presence of multiple alternate pathways between glutamate and succinate. The conversion of glutamate to succinate via $\gamma$-aminobutyrate and $\gamma$-glutamylsuccinate showed similar flux ranges as AKGDH indicating the inability of 13C-MFA to resolve between these alternative pathways. Another pathway contributing to the expanded AKGDH range was the degradation of arginine which had a non-zero lower bound to account for the production of biomass components, putrescine and spermidine. Expansion of FUM and MDH flux ranges were due to the presence of amino group transferring mechanisms in the arginine and purine biosynthetic pathways which remove the amino group from aspartate to produce fumarate. The lower bound of MDH was as low as zero due to the presence of an alternate MDH (MQO) which uses ubiquinone as the electron acceptor. Despite the presence of wide ranges at the individual flux level, the sum total of all alternate pathways for a particular reaction resulted in similar bounds as in the core model. The glyoxylate shunt was equally well resolved in the GSMM model as in the core one but with an expanded repertoire of functions. The production of glycoaldehyde as a by-product of tetrahydrofolate biosynthesis and the eventual conversion of glycoaldehyde to glyoxylate resulted in a partial glyoxylate shunt activity in which MALS was active with a non-zero lower bound. 


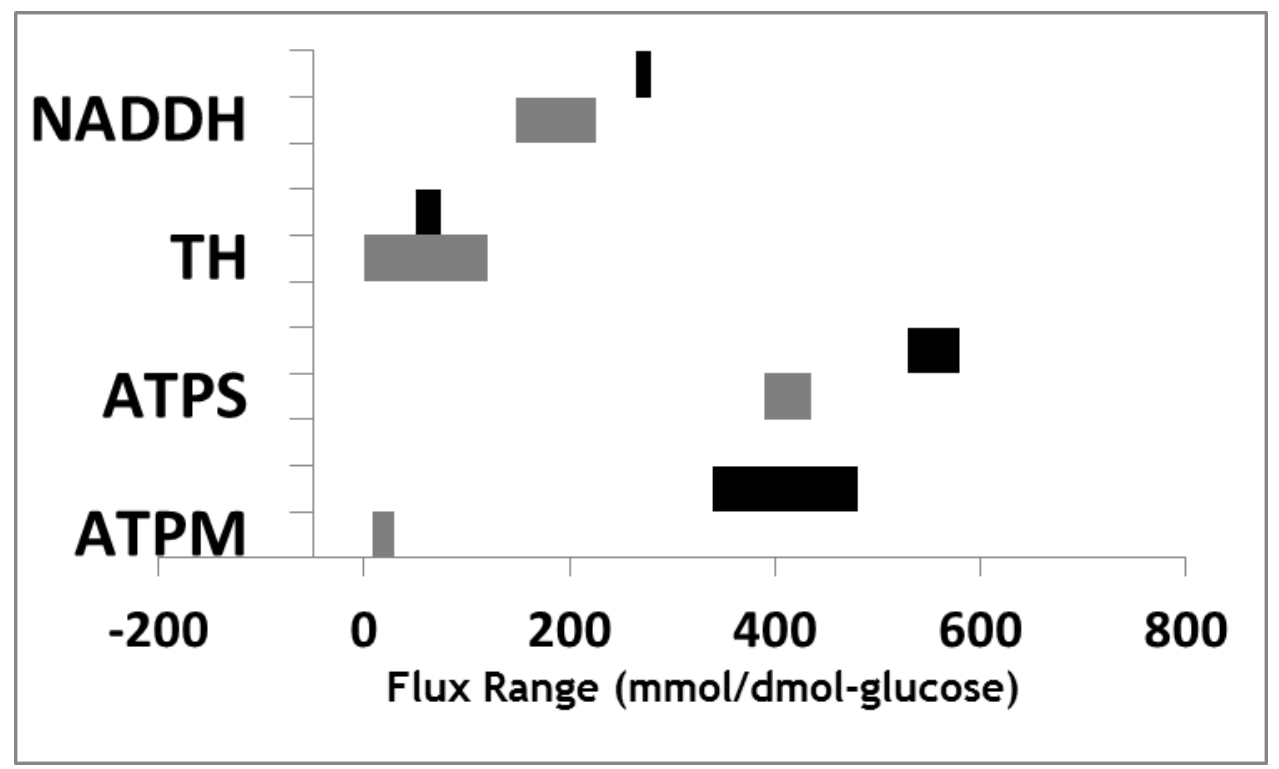

Figure 3: Resolution of energy metabolism in ( $\bullet$ ) core model and (-) GSM model.

Reactions that were insensitive to the C13 labels and thus outside EMU balances are indirectly resolved by component balance limitations imposed by flux ranges of EMU-resolved reactions. Such reactions include a majority of cofactor biosynthesis pathways, lipid biosynthesis, pyrimidine biosynthesis, and energy metabolism. Figure 3 shows the flux ranges corresponding the oxidative phosphorylation, NADH transhydrogenase, and total free ATP within the network. Oxidative phosphorylation was well resolved as the oxygen uptake limits total flux through this pathway. The presence of pathways that convert NADPH to NADH at the expense of ATP results in a finite upper bound for the transhydrogenase due to ATP limits. A negative lower bound for this reaction indicates that the direction of this reaction could not be resolved by $13 \mathrm{C}$ MFA. The lower bound of ATPM is 8.39, which matches exactly the non-growth associated ATP maintenance requirement in iAF1260. The upper bound of available ATP predicted using the GSMM model was far lower than that of the core model due to the fact that the GSM model globally accounts for all ATP requirements. The flux range of excess ATP availability predicted using the GSM model was far less than the core model due to the fact that the core model only accounts for quantifiable (i.e., polymerization and biosynthetic of macromolecules) ATP costs, which only amounts to $56 \%$ of the total growth-associated requirement (Feist et al., 2007). A side product of the well resolved ATP balance is that the activity of futile cycles is highly constrained and can thus be resolved by FVA. 
Reaction flux resolution by $13 \mathrm{C}$-MFA requires that there exist distinct atom transition profiles between alternatives. This property affects the resolvability of gluconeogenesis, ED pathway and the glyoxylate shunt. The flux through gluconeogenesis can be estimated by the intersection of the ranges of three reactions: PPS, FBP, and G6PP which reverse reactions PYK, PFK, and HK, respectively from glycolysis. PFK alone provides a sharp estimate for gluconeogenesis as it is the only well resolved flux out of the three. G6PP is unresolvable despite the fact that glucose-6phosphate and glucose have distinct labeling patterns because the labeling pattern of intracellular glucose is not measured. In addition, an inactive malic enzyme results in identical phosphoenolpyruvate and pyruvate labeling patterns thereby rendering PPS unresolvable. The reversibility of TPI and FBA result in altered labeling patterns of fructose-1,6-bisphosphate compared to fructose-6-phosphate. An active FBP would alter the labeling pattern of all metabolites within the PPP thereby affecting the observed labeling patterns of downstream amino acids such as glycine, serine, alanine, valine, leucine, and isoleucine. This property aids in the resolution of FBP and thus facilitating the resolution of gluconeogenesis to within $8 \%$ of the total glucose uptake. No information regarding the activity of gluconeogenesis can be gleaned using only the core model. The ED pathway is equally well resolved using the GSMM as in the core model due to the fact that it produces a pyruvate molecule with a different carbon atom arrangement compared to glycolysis. This directly impacts the predicted labeling of alanine and the branched chain amino acids derived from pyruvate. Similarly, flux through the glyoxylate shunt produces a differently labeled aspartate when compared to the conventional TCA pathway.

A closer analysis of the obtained flux ranges provided an insight into the sensitivity of the obtained flux ranges to biomass composition. The biomass composition and the necessary drains from central metabolism are shown in supplementary Table S2. Any perturbations to drains from central metabolism lie within the estimated $95 \%$ confidence interval of all the fluxes thereby rendering flux predictions insensitive to perturbed biomass composition assuming that the range of perturbation does not exceed $10 \%$. It was found that the size of the obtained flux ranges was primarily due to errors in extracellular flux measurements. To confirm this, we re-estimated the fluxes and flux ranges for the same network while allowing a $10 \%$ change to each biomass component individually while maintaining the cell dry weight. The absence of any significant flux range shift, along with a proportional increase to the drain of our target biomass component from central metabolism corresponding to perturbation confirmed our hypothesis of insensitivity 
of flux ranges to biomass composition perturbation given this data set. Even though fluxes of biomass components changed in proportion to the perturbation, any additional impact on central metabolism was minimal.

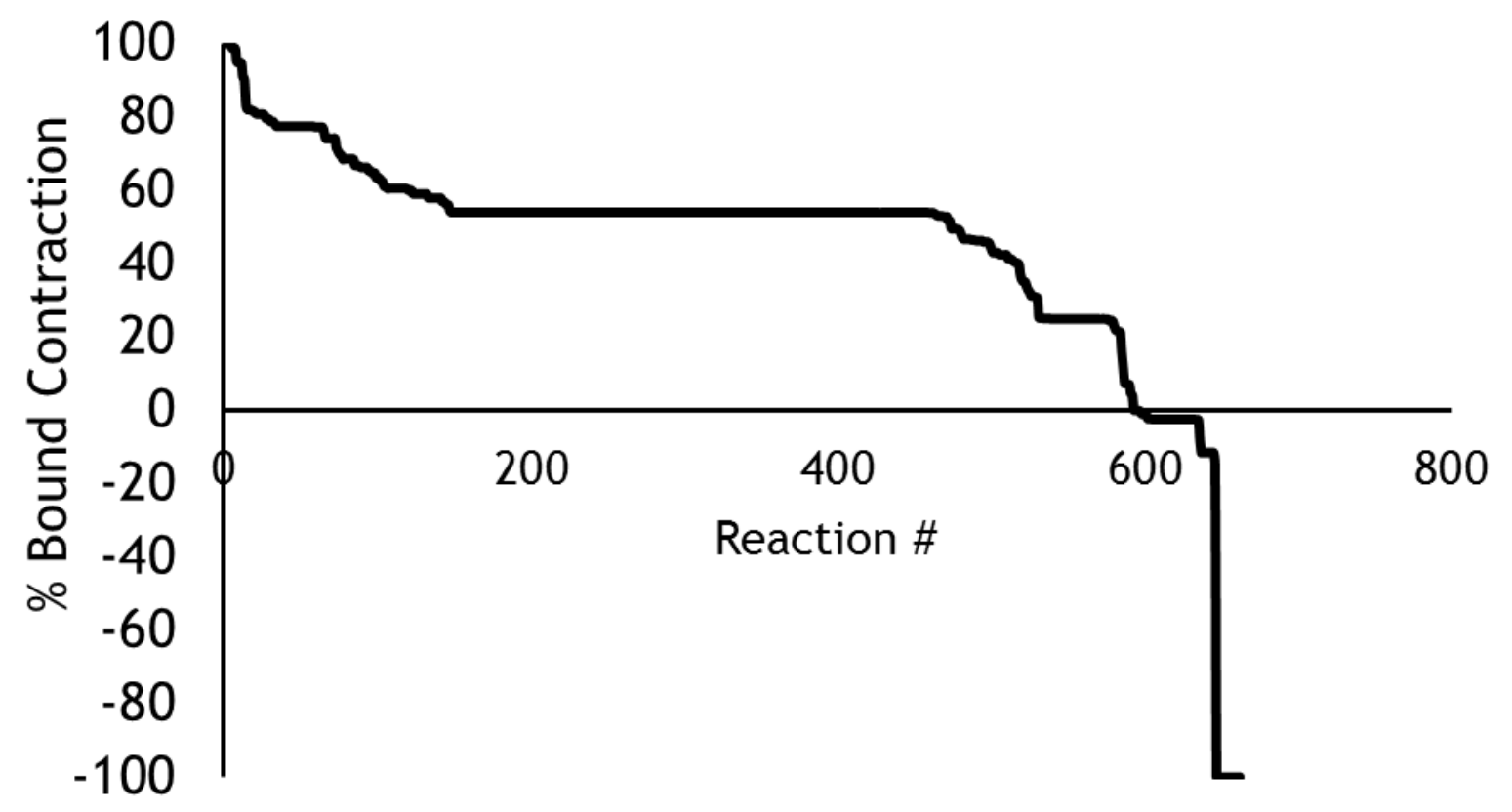

Figure 4: Loss of information expressed as \% bound contraction of flux ranges for every reaction in the GSM model when flux ranges are estimated using FVA with core model-based MFA derived flux ranges as constraints.

As described earlier a common practice is to perform MFA using a core model and then project the identified flux ranges onto a genome-scale model. We performed the same two-stage implementation and compared the results with MFA using the full GSMM model. We found that the use of the core model for MFA generates flux ranges that upon mapping onto the GSM model, propagate all assumptions made during the construction of the core model. For as many as $90 \%$ of the reactions in the GSM flux ranges are more restricted than when the full GSMM is used for MFA. Figure 4 shows the distribution of flux range contraction upon imposing flux ranges derived for the core model to obtain GSM flux ranges using FVA. For more than half of the reactions in the GSM model, ranges are more than halved compared to the correct elucidation 
using the GSMM model. Notably, there are 295 reactions whose estimated range upon projection to the GSM model was $54 \%$ narrower than supported by the data. This demonstrates in a quantitative manner the fact that assumptions made during the core model construction propagate onto the GSM model leading to possibly erroneous conclusions about reaction flux identifiability. Overly tight flux ranges predicted using the core model often shut down alternate pathways which confounds reaction essentiality prediction. For example, acetate kinase is essential based on predictions using core model MFA, however, using a GSMM based MFA the reaction is correctly predicted (Baba et al., 2006) as non-essential. Acetate production can be taken over by the POX reaction which generates ATP by transferring electrons from pyruvate to the electron transport chain while producing acetate and carbon dioxide. In contrast, for about $10 \%$ of reactions MFA using the GSMM model leads to tighter flux resolution compared to the core model. These reactions include energy balance reactions such as oxidative phosphorylation which requires network-wide resolution of redox balances for proper resolution. This quantitatively demonstrates the significance of describing metabolism at the genome-scale and the feasibility of inferring fluxes using MFA at a genome-scale.

\section{DISCUSSION}

In this paper, we have applied the framework of 13C-MFA to perform flux and range elucidation using a genome-scale model. Using available extracellular flux measurement data, we eliminated blocked reactions and those incapable of carrying flux in the iAF1260 GSM model of E.coli using FVA. The resulting GSM model contained 697 reactions and 595 metabolites with 29 reversible reactions. The corresponding atom mapping model was generated by integrating the atom mapping information for every reaction in this GSM model using the CLCA algorithm. Finally, the network was decomposed using the EMU algorithm so as to evaluate MIDs of target metabolite fragments for a given flux distribution, so as to iteratively obtain an optimal flux distribution which best explains experimentally observed GCMS labeling data. In order to account for degeneracy within the metabolic network and the inherent error associated with experimental data, we also evaluated the $95 \%$ confidence intervals associated with each flux. Given the computationally intensive nature of the 13C-MFA procedure, we modified the confidence interval estimation procedure so as to decrease the computational time by up to $67 \%$ by identifying the minimal set of fluxes whose $95 \%$ confidence intervals need to be evaluated 
using flux coupling analysis checks. We also redefined the definition of $\chi^{2}$ degrees of freedom to better describe the structural properties of the genome-scale EMU model and found that our obtained optimal flux distribution was statistically acceptable.

We found that the GSM model is able to produce a better fit compared to the core model owing to improved prediction of alanine and valine MS data. While the overall flux distribution remained similar to that of the core model, the comprehensive biomass equation used in the GSM model resulted in a shifted PPP range. The presence of gluconeogenesis along with glycolysis created futile cycles for three key reactions, of which only PFK could be resolved properly using 13C-MFA. The availability of the methylglyoxal pathway as an alternative to lower glycolysis resulted in a reduction of the lower bound for TPI, GAPD, PGK, and ENO. PYK experienced significant bound expansion in the GSM model compared to the core model due to the availability of the PTS mechanism for glucose uptake as an alternate pathway facilitating the conversion of phosphoenolpyruvate to pyruvate. The reduced flux through PDH was found to be due to the activity of POX, which is known to be a key reaction in the growth of E.coli under aerobic condition (Abdel-Hamid et al., 2001; Li et al., 2007). The expansion in the TCA cycle ranges was due to increased glutamate synthesis for biomass production, availability of arginine and glutamate degradation as alternatives to $\mathrm{AKGDH}$, and the presence of an additional amino group donation mechanism with aspartate as the donor and fumarate as the product. Since both evaluated tracer schemes produced consistent results, it is evident that the inability to resolve certain pathways is not tracer-specific (Figure 2 and Supplementary figure 1), but a property of the GSMM model, indicating the need for additional experimental data to completely resolve all fluxes in the metabolic network. We found that energy metabolism was quite well resolved with the amount of free ATP being greatly limited thereby facilitating the resolution of futile cycles. The transhydrogenase reaction, on the other hand was poorly resolved with much uncertainty in its predicted direction. Finally, we found that utilization of the bounds estimated using the core model resulted in much smaller flux ranges in as many as $90 \%$ of the reactions due to the fact that these bounds carry with them the assumptions involved in the creation of the core model. The source of this reduction was found to be the inactivation of alternate pathways such as glutamate degradation, and futile cycles such as gluconeogenesis, occurring due to tighter mass balance constraints imposed by the flux ranges estimated using the core model. While such assumptions do accelerate flux computations by elimination of variables 
and provide information on total sum of fluxes through all alternate routes for a given transformation, they fail to account for flexibility within the network, thus having an adverse impact on secondary inferences from the estimated flux ranges. As such, this study highlights the need for the use of more comprehensive models for flux elucidation so as to obtain a better agreement with experimentally observed data and improved quality of inference. The flux range expansion cannot be resolved by optimal tracer design alone because it stems from the presence of alternate pathways with identical overall atom transitions. This loss of resolution will persist even if every branch point were to be resolved perfectly (e.g., by COMPLETE-MFA). Therefore, flux and range estimation using alternate glucose-based tracer schemes (Crown et al., 2015) or simultaneous fitting of multiple data sets (Leighty and Antoniewicz, 2013) would be unable to resolve this ambiguity in alternate pathway activity, as confirmed by the evaluation of multiple tracer schemes in this study.

A major challenge with MFA using GSM models is the increase in computation time associated with the vast increase in the number of variables. An effective way to address this issue would be to simplify the genome-scale MFA model to the size of a (near) core model. The strategy here is to decrease the number of variables while retaining all the information regarding novel mappings and alternate pathways contained within the GSM model. An elementary reduction method for simplification of linear pathways at the EMU network level has already been proposed (Antoniewicz et al., 2007a), but not yet implemented. Analysis of the EMU model has already revealed that only $60 \%$ of all the reactions contained within the GSM model can be resolved using 13C-MFA. We have also seen that the actual regression model described by EMU balances has only 99 free variables. As such, the decoupling of the EMU network from the entire GSM model alone can result in a $63 \%$ reduction in the number of free fluxes. Further reduction of EMU networks can be achieved by elimination of linear pathways, so as to obtain the simplest possible EMU network of each size. The reduction of model size will help alleviate some of the complexity associated with inclusion of intracellular compartments.

Better resolution of fluxes using a GSM model requires an optimal tracer design and the maximum set of extracellular flux measurements. It has already been shown that no single tracer is sufficient to resolve all the fluxes within a metabolic network, and that different tracers promote the resolution of specific branch points (Leighty and Antoniewicz, 2013). While MFA 
using multiple data sets does work effectively on a core model, it faces the same problems associated with scale-up at the genome-scale. This can be overcome by designing an isotopelabeling experiment using an optimal set of tracers and GCMS measurements, which can reliably resolve all the key branch points in the metabolic network. The basis for an optimal tracer design has already been proposed in the form of EMU basis vectors (Crown and Antoniewicz, 2012), which decouples substrate labeling from the fluxes in the model. Trade-offs between cost of the tracer and its resolving power are often an important consideration in MFA. Note that small differences in PPP, ED, ME and TCA cycle flux elucidation quality between tracers 2-13C and 5-13C glucose using a core model (Leighty and Antoniewicz, 2013) disappear when performing MFA at a genome-scale (see Supplementary Table 1). This is because the presence of additional drains and alternate pathways in the GSM model widen the inferred flux ranges thus overwhelming any gains afforded by the tracer choice. The resolution fidelity of the remaining flux ranges in the GSM model remain unaffected by tracer choice alluding to the significant role played by stoichiometric constraints and flux coupling analysis in flux elucidation. This implies that even sub-optimal lower cost alternatives such as the widely used 4:1 mixture of 1-13C and U-13C (\$217/gram) glucose (Crown et al., 2015) may be used to obtain reliable genome-scale flux resolution in place of more expensive tracers such as 2-13C (\$585/gram) or 5-13C (\$1700/gram) glucose (http://www.sigmaaldrich.com). The availability of MS measurements for other metabolites besides amino acids will further improve the resolution of poorly resolved fluxes in the GSM model. MS measurements of fatty acids (Yoo et al., 2008) and intracellular metabolites (Luo et al., 2007; Metallo et al., 2012) have already been utilized to infer flux distributions. In regards to the flux elucidation presented in this paper, Table 2 provides a candidate list of metabolites that if measured would resolve alternate routes. This is based on the idea that, if the labeling distribution of a metabolite unique to a given alternate pathway is different from the labeling distribution at the start of the experiment then the pathway carries flux and the steady-state flux through the pathway can be evaluated using an isotopic nonstationary flux analysis procedure for E. coli as described previously (Noh et al., 2007; Young et al., 2008). Noh et al (2007) have used a rapid sampling approach followed by methanol quenching to obtain multiple isotopic non-stationary data points before attainment of isotopic steady-state. Pool size measurement using already established protocols for methylglyoxal (Girgis et al., 2012) and $\gamma$-aminobutyrate (O'Byrne et al., 2011) are already available for 
resolving flux through their corresponding pathways. Incorporation of these additional measurements can further sharpen the estimated flux ranges to provide a better resolution of metabolism. The estimated flux ranges can be refined further with the availability of a more complete set of extracellular flux measurements to include secretion profiles of additional products, such as succinate and other organic acids, so as to close the carbon balance for the given growth condition. These set of measurements will reduce the solution space by constraining the flux through various production pathways, thereby further simplifying the task of identifying the optimal flux distribution. A complete set of measurements required for maximum flux elucidation can be obtained using a formulation such as OptMeas (Chang et al., 2008).

Table 2: Additional suggested MS measurements for resolving various alternate routes.

\begin{tabular}{|cccc|}
$\begin{array}{c}\text { Central } \\
\text { Metabolic } \\
\text { pathway }\end{array}$ & $\begin{array}{c}\text { Alternate } \\
\text { Pathway }\end{array}$ & $\begin{array}{c}\text { Metabolite } \\
\text { measurement } \\
\text { candidate }\end{array}$ & $\begin{array}{c}\text { Measurement } \\
\text { Type }\end{array}$ \\
\hline Lower glycolysis & $\begin{array}{c}\text { Methylglyoxal } \\
\text { pathway }\end{array}$ & methylglyoxal & $\begin{array}{c}\text { Time-course MS } \\
\text { with known pool } \\
\text { size }\end{array}$ \\
TCA cycle & Arginine & -aminobutyrate & $\begin{array}{c}\text { Time-course MS } \\
\text { with known pool } \\
\text { size }\end{array}$ \\
degradation & G6PP & $\begin{array}{c}\text { Intracellular } \\
\text { glucose }\end{array}$ & Steady-state MS \\
cycle & & & \\
\hline
\end{tabular}

The extent of resolution obtained using a GSM model for E.coli points to the possibility of more reliable flux elucidation and biologically relevant inferences in more complex systems such as yeast, plants, and mammalian systems with compartmentalized metabolism. Application of genome-scale MFA to such systems will allow the use of closed cofactor balances without the risk of altering the actual flux distribution predicted using the flux estimation procedure. This will enable identifying metabolic bottlenecks leading to more informed metabolic engineering interventions that improve the yield of target products. It is important to note that flux resolution using 13C-MFA ultimately depends on how well the organism's genome is annotated, the complexity of the underlying EMU network, and the quality of experimental data used for flux estimation. 


\section{Acknowledgement}

This work was supported by the U.S. Department of Energy (DOE) at the Pennsylvania State University, University Park, under Grant DE-SC10822882. 


\section{Appendix A}

\section{Predicting labeling patterns}

Decomposing the network using the EMU algorithm provides an exhaustive list of metabolite fragments and reactions involved in predicting the labeling pattern of target metabolite fragments for a given tracer input and flux distribution. The mass balance for a reaction within the EMU network at isotopic and metabolic steady state shown below is described as:

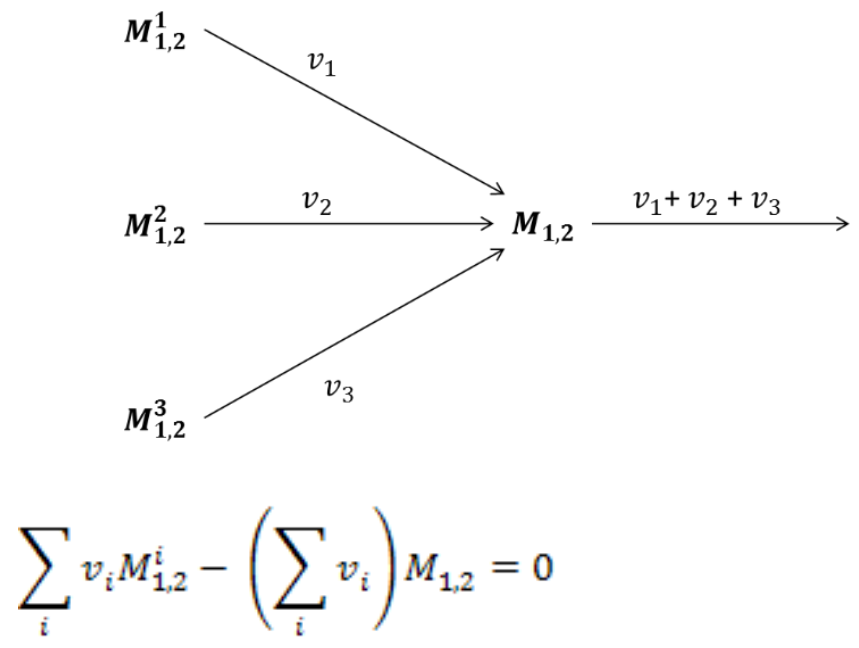

If $\mathrm{M}^{3}$ is a substrate to the network, then the above equation can be re-written as,

$$
\sum_{i=1,2} v_{i} M_{1,2}^{i}-\left(\sum_{i} v_{i}\right) M_{1,2}=-v_{3} M_{1,2}^{3}
$$

Based on equations (1) and (2), the mass balance for all the reactions of a particular EMU size can be expressed as:

$$
A X=B Y
$$

Where, $\mathbf{X}$ and $\mathbf{Y}$ represent the vectors of balanced and input EMUs respectively, and, A, and $\mathbf{B}$ are the corresponding coefficient matrices, which are functions of fluxes. Since $\mathbf{A}$ is a square matrix, $\mathbf{X}$ can be solved by inversion of $\mathbf{A}$ and multiplying it with the r.h.s. of equation (3). The set of target metabolite fragments, $\mathbf{x}$, is a subset of $\mathbf{X}$, and their corresponding mass isotopomer 
distributions (MIDs) can be obtained by solving equation (3). The MIDs estimated above need to be corrected for uncorrected pool dilutions, and additional label dilution arising from sparged $\mathrm{CO}_{2}$ (Leighty and Antoniewicz, 2012; Leighty and Antoniewicz, 2013).

\section{Least-Squares NLP}

$$
\begin{gathered}
\min _{v} \sum_{i=1}^{N}\left(\frac{x(v)_{i}^{p}-x_{i}^{m}}{\sigma_{i}}\right)^{2} \\
\text { s.t. } \quad \text { S. } v=0 \\
v_{j}^{L B} \leq v_{j} \leq v_{j}^{U B}
\end{gathered}
$$

Where,

$x(v)_{i}^{p}$ is the predicted labeling pattern of fragment $I$ for a given flux distribution, $\mathbf{v}$.

$x_{\tilde{i}}^{m}$ is the experimentally measured labeling pattern for fragment $\mathrm{i}$.

$\sigma_{i}$ is the standard error of measurement for fragment $\mathrm{i}$.

$\mathrm{S}$ is the stoichiometry matrix.

$v_{j}^{L B}$ is the lower bound on flux $\mathrm{v}_{\mathrm{j}}$

$v_{j}^{U B}$ is the upper bound on flux $\mathrm{v}_{\mathrm{j}}$.

\section{Implementation}

The equality constraint in the above formulation can be eliminated by expressing the vector of fluxes, $\mathbf{v}$, in terms of the set of free fluxes, $\mathbf{u}$, using:

$v=N \cdot u$

Where, $\mathrm{N}$ is the rational basis for the null space of $\mathrm{S}$.

Since a non-negativity constraint is imposed on all fluxes, the equality constraint can be replaced with the inequality constraint:

$N . u \geq 0$ 
If bounds for $\mathbf{v}$ are available, the above inequality constraint can be modified to account for the lower and upper bounds, $\mathbf{v}^{\mathrm{LB}}$, and $\mathbf{v}^{\mathrm{UB}}$ :

$N \cdot u \geq v^{L B}$

$N . u \leq v^{U B}$

This transforming the NLP problem to:

$$
\begin{gathered}
\min _{u} \sum_{i=1}^{N}\left(\frac{x(\boldsymbol{u})_{i}^{p}-x_{i}^{m}}{\sigma_{i}}\right)^{2} \\
\text { s.t. } \quad \boldsymbol{N} . \boldsymbol{u} \geq v^{L B} \\
\quad \boldsymbol{N} \cdot \boldsymbol{u} \leq \boldsymbol{v}^{U B}
\end{gathered}
$$

The above minimization problem can be solved using the fmincon function within the Optimization Toolbox in MATLAB ${ }^{\mathrm{TM}}$. Among the different algorithm options, the interior-point algorithm accepts a user-supplied Hessian, which can be computed using the first-order Taylor Series expansion(Antoniewicz et al., 2006) of the objective function to yield:

$$
H=\left(\frac{d \boldsymbol{x}}{d \boldsymbol{u}}\right)^{T} W^{-1}\left(\frac{d \boldsymbol{x}}{d \boldsymbol{u}}\right)
$$

Where $\mathrm{W}$ is the covariance matrix serving as a weighting matrix for the least-squares minimization problem.

The derivative of the estimated MIDs with respect to the free fluxes can be estimated using the following equation.

$$
\begin{gathered}
\left(\frac{d x}{d u}\right)^{T}=\left(\frac{d x}{d v}\right)^{T} *\left(\frac{d v}{d u}\right) \\
\left(\frac{d x}{d u}\right)^{T}=\left(\frac{d x}{d v}\right)^{T} * N
\end{gathered}
$$

To obtain the derivative of MIDs with respect to all the fluxes within the network, we have to differentiate equation (3), and rearrange it to obtain the following expression.

$$
\frac{d \boldsymbol{X}}{d \nu}=A^{-1}\left(\frac{d B}{d v} * Y+B * \frac{d Y}{d v}\right)
$$

\section{Estimation of confidence intervals}


With the EMU network spanning a much smaller portion of the overall metabolic network, the original procedure for estimation of confidence intervals was modified to enhance the speed of estimation. While the range estimation procedure(Antoniewicz et al., 2006) of an individual flux was not modified, the set of fluxes whose ranges need to be directly determined was reduced based on flux coupling properties. The procedure is as follows:

Step 1: $\quad$ Define the initial set of fluxes ( $\mathbf{v})$ as the union of the sets of fluxes involved in EMU balances and those corresponding to an extracellular measured flux.

Step 2: $\quad$ Identify all fluxes coupled to an extracellular measurement and eliminate these fluxes from $\mathbf{v}$.

Step 3: $\quad$ For each flux, $v_{\mathrm{i}}$, within $\mathbf{v}$, identify and eliminate fluxes, $\mathrm{v}_{\mathrm{j}}$, that are fully coupled to $\mathrm{vi}$.

Step 4: $\quad$ Estimate the $95 \%$ confidence interval for all the fluxes remaining in $\mathbf{v}$.

Step 5: Using the estimated confidence intervals as flux bounds, perform an FVA to estimate all the other flux ranges within the metabolic network.

While performing FVA, only the net flux of reversible reactions was considered due to the fact that exchange fluxes which are not involved in EMU balances cannot be resolved by 13C-MFA. 


\section{REFERENCES}

Abdel-Hamid, A. M., Attwood, M. M., Guest, J. R., 2001. Pyruvate oxidase contributes to the aerobic growth efficiency of Escherichia coli. Microbiology. 147, 1483-98.

Ahn, W. S., Antoniewicz, M. R., 2011. Metabolic flux analysis of $\mathrm{CHO}$ cells at growth and non-growth phases using isotopic tracers and mass spectrometry. Metabolic engineering. 13, 598-609.

Antoniewicz, M. R., Kelleher, J. K., Stephanopoulos, G., 2006. Determination of confidence intervals of metabolic fluxes estimated from stable isotope measurements. Metabolic engineering. 8, 32437.

Antoniewicz, M. R., Kelleher, J. K., Stephanopoulos, G., 2007a. Elementary metabolite units (EMU): a novel framework for modeling isotopic distributions. Metabolic engineering. 9, 68-86.

Antoniewicz, M. R., Kraynie, D. F., Laffend, L. A., Gonzalez-Lergier, J., Kelleher, J. K., Stephanopoulos, G., 2007b. Metabolic flux analysis in a nonstationary system: fed-batch fermentation of a high yielding strain of E. coli producing 1,3-propanediol. Metabolic engineering. 9, 277-92.

Baba, T., Ara, T., Hasegawa, M., Takai, Y., Okumura, Y., Baba, M., Datsenko, K. A., Tomita, M., Wanner, B. L., Mori, H., 2006. Construction of Escherichia coli K-12 in-frame, single-gene knockout mutants: the Keio collection. Molecular systems biology. 2, 20060008.

Bonarius, H. P., Timmerarends, B., de Gooijer, C. D., Tramper, J., 1998. Metabolite-balancing techniques vs. $13 \mathrm{C}$ tracer experiments to determine metabolic fluxes in hybridoma cells. Biotechnology and bioengineering. 58, 258-62.

Burgard, A. P., Nikolaev, E. V., Schilling, C. H., Maranas, C. D., 2004. Flux coupling analysis of genomescale metabolic network reconstructions. Genome research. 14, 301-12.

Byrd, R. H., Gilbert, J. C., Nocedal, J., 2000. A trust region method based on interior point techniques for nonlinear programming. Math. Program. 89, 149-185.

Byrd, R. H., Hribar, M. E., Nocedal, J., 1999. An Interior Point Algorithm for Large-Scale Nonlinear Programming. SIAM J. on Optimization. 9, 877-900.

Chang, Y., Suthers, P. F., Maranas, C. D., 2008. Identification of optimal measurement sets for complete flux elucidation in metabolic flux analysis experiments. Biotechnology and bioengineering. 100, 1039-49.

Chen, X., Alonso, A. P., Allen, D. K., Reed, J. L., Shachar-Hill, Y., 2011. Synergy between (13)C-metabolic flux analysis and flux balance analysis for understanding metabolic adaptation to anaerobiosis in E. coli. Metabolic engineering. 13, 38-48.

Copeland, W. B., Bartley, B. A., Chandran, D., Galdzicki, M., Kim, K. H., Sleight, S. C., Maranas, C. D., Sauro, H. M., 2012. Computational tools for metabolic engineering. Metabolic engineering. 14, 270-80.

Crown, S. B., Antoniewicz, M. R., 2012. Selection of tracers for 13C-metabolic flux analysis using elementary metabolite units (EMU) basis vector methodology. Metabolic engineering. 14, 15061.

Crown, S. B., Indurthi, D. C., Ahn, W. S., Choi, J., Papoutsakis, E. T., Antoniewicz, M. R., 2011. Resolving the TCA cycle and pentose-phosphate pathway of Clostridium acetobutylicum ATCC 824: Isotopomer analysis, in vitro activities and expression analysis. Biotechnology journal. 6, 300-5.

Crown, S. B., Long, C. P., Antoniewicz, M. R., 2015. Integrated 13C-metabolic flux analysis of 14 parallel labeling experiments in Escherichia coli. Metabolic engineering. 28, 151-8. 
Dash, S., Mueller, T. J., Venkataramanan, K. P., Papoutsakis, E. T., Maranas, C. D., 2014. Capturing the response of Clostridium acetobutylicum to chemical stressors using a regulated genome-scale metabolic model. Biotechnology for biofuels. 7, 144.

Feist, A. M., Henry, C. S., Reed, J. L., Krummenacker, M., Joyce, A. R., Karp, P. D., Broadbelt, L. J., Hatzimanikatis, V., Palsson, B. O., 2007. A genome-scale metabolic reconstruction for Escherichia coli K-12 MG1655 that accounts for 1260 ORFs and thermodynamic information. Molecular systems biology. 3, 121.

Flores, S., Gosset, G., Flores, N., de Graaf, A. A., Bolivar, F., 2002. Analysis of carbon metabolism in Escherichia coli strains with an inactive phosphotransferase system by (13)C labeling and NMR spectroscopy. Metabolic engineering. 4, 124-37.

Girgis, H. S., Harris, K., Tavazoie, S., 2012. Large mutational target size for rapid emergence of bacterial persistence. Proceedings of the National Academy of Sciences of the United States of America. 109, 12740-12745.

Holms, H., 1996. Flux analysis and control of the central metabolic pathways in Escherichia coli. FEMS microbiology reviews. 19, 85-116.

Hua, Q., Yang, C., Baba, T., Mori, H., Shimizu, K., 2003. Responses of the central metabolism in Escherichia coli to phosphoglucose isomerase and glucose-6-phosphate dehydrogenase knockouts. Journal of bacteriology. 185, 7053-67.

Korner, R., Apostolakis, J., 2008. Automatic determination of reaction mappings and reaction center information. 1. The imaginary transition state energy approach. Journal of chemical information and modeling. 48, 1181-9.

Kumar, A., Maranas, C. D., 2014. CLCA: maximum common molecular substructure queries within the MetRxn database. J Chem Inf Model. 54, 3417-38.

Kumar, A., Suthers, P. F., Maranas, C. D., 2012. MetRxn: a knowledgebase of metabolites and reactions spanning metabolic models and databases. BMC bioinformatics. 13, 6.

Latendresse, M., Malerich, J. P., Travers, M., Karp, P. D., 2012. Accurate atom-mapping computation for biochemical reactions. J Chem Inf Model. 52, 2970-82.

Leighty, R. W., Antoniewicz, M. R., 2012. Parallel labeling experiments with [U-13C]glucose validate E. coli metabolic network model for $13 \mathrm{C}$ metabolic flux analysis. Metabolic engineering. 14, 53341.

Leighty, R. W., Antoniewicz, M. R., 2013. COMPLETE-MFA: complementary parallel labeling experiments technique for metabolic flux analysis. Metabolic engineering. 20, 49-55.

Li, M., Yao, S., Shimizu, K., 2007. Effect of poxB gene knockout on metabolism in Escherichia coli based on growth characteristics and enzyme activities. World J Microbiol Biotechnol. 23, 573-580.

Long, C. P., Antoniewicz, M. R., 2014. Quantifying biomass composition by gas chromatography/mass spectrometry. Analytical chemistry. 86, 9423-7.

Luo, B., Groenke, K., Takors, R., Wandrey, C., Oldiges, M., 2007. Simultaneous determination of multiple intracellular metabolites in glycolysis, pentose phosphate pathway and tricarboxylic acid cycle by liquid chromatography-mass spectrometry. Journal of chromatography. A. 1147, 153-64.

Mahadevan, R., Schilling, C. H., 2003. The effects of alternate optimal solutions in constraint-based genome-scale metabolic models. Metabolic engineering. 5, 264-76.

Metallo, C. M., Gameiro, P. A., Bell, E. L., Mattaini, K. R., Yang, J., Hiller, K., Jewell, C. M., Johnson, Z. R., Irvine, D. J., Guarente, L., Kelleher, J. K., Vander Heiden, M. G., Iliopoulos, O., Stephanopoulos, G., 2012. Reductive glutamine metabolism by IDH1 mediates lipogenesis under hypoxia. Nature. 481, 380-4.

Mollney, M., Wiechert, W., Kownatzki, D., de Graaf, A. A., 1999. Bidirectional reaction steps in metabolic networks: IV. Optimal design of isotopomer labeling experiments. Biotechnology and bioengineering. 66, 86-103. 
Murphy, T. A., Dang, C. V., Young, J. D., 2013. Isotopically nonstationary 13C flux analysis of Myc-induced metabolic reprogramming in B-cells. Metabolic engineering. 15, 206-17.

Nielsen, J., 2003. It is all about metabolic fluxes. Journal of bacteriology. 185, 7031-5.

Noh, K., Gronke, K., Luo, B., Takors, R., Oldiges, M., Wiechert, W., 2007. Metabolic flux analysis at ultra short time scale: isotopically non-stationary $13 \mathrm{C}$ labeling experiments. Journal of biotechnology. 129, 249-67.

O'Byrne, C. P., Feehily, C., Ham, R., Karatzas, K. A., 2011. A modified rapid enzymatic microtiter plate assay for the quantification of intracellular gamma-aminobutyric acid and succinate semialdehyde in bacterial cells. J Microbiol Methods. 84, 137-9.

Pazman, A., 1993. Nonlinear statistical models.

Saha, R., Verseput, A. T., Berla, B. M., Mueller, T. J., Pakrasi, H. B., Maranas, C. D., 2012. Reconstruction and comparison of the metabolic potential of cyanobacteria Cyanothece sp. ATCC 51142 and Synechocystis sp. PCC 6803. PloS one. 7, e48285.

Schmidt, K., Carlsen, M., Nielsen, J., Villadsen, J., 1997. Modeling isotopomer distributions in biochemical networks using isotopomer mapping matrices. Biotechnology and bioengineering. $55,831-40$.

Schmidt, K., Nielsen, J., Villadsen, J., 1999. Quantitative analysis of metabolic fluxes in Escherichia coli, using two-dimensional NMR spectroscopy and complete isotopomer models. Journal of biotechnology. 71, 175-89.

Segre, D., Vitkup, D., Church, G. M., 2002. Analysis of optimality in natural and perturbed metabolic networks. Proceedings of the National Academy of Sciences of the United States of America. 99, 15112-7.

Shimizu, K., 2004. Metabolic flux analysis based on 13C-labeling experiments and integration of the information with gene and protein expression patterns. Advances in biochemical engineering/biotechnology. 91, 1-49.

Suthers, P. F., Burgard, A. P., Dasika, M. S., Nowroozi, F., Van Dien, S., Keasling, J. D., Maranas, C. D., 2007. Metabolic flux elucidation for large-scale models using $13 \mathrm{C}$ labeled isotopes. Metabolic engineering. 9, 387-405.

Usui, Y., Hirasawa, T., Furusawa, C., Shirai, T., Yamamoto, N., Mori, H., Shimizu, H., 2012. Investigating the effects of perturbations to pgi and eno gene expression on central carbon metabolism in Escherichia coli using (13)C metabolic flux analysis. Microbial cell factories. 11, 87.

van Gulik, W. M., Heijnen, J. J., 1995. A metabolic network stoichiometry analysis of microbial growth and product formation. Biotechnology and bioengineering. 48, 681-98.

Varma, A., Palsson, B. O., 1994. Stoichiometric flux balance models quantitatively predict growth and metabolic by-product secretion in wild-type Escherichia coli W3110. Applied and environmental microbiology. 60, 3724-31.

Waltz, R. A., Morales, J. L., Nocedal, J., Orban, D., 2006. An interior algorithm for nonlinear optimization that combines line search and trust region steps. Math. Program. 107, 391-408.

Wiechert, W., de Graaf, A. A., 1996. In vivo stationary flux analysis by $13 \mathrm{C}$ labeling experiments. Advances in biochemical engineering/biotechnology. 54, 109-54.

Wiechert, W., Mollney, M., Isermann, N., Wurzel, M., de Graaf, A. A., 1999. Bidirectional reaction steps in metabolic networks: III. Explicit solution and analysis of isotopomer labeling systems. Biotechnology and bioengineering. 66, 69-85.

Wiechert, W., Siefke, C., de Graaf, A. A., Marx, A., 1997. Bidirectional reaction steps in metabolic networks: II. Flux estimation and statistical analysis. Biotechnology and bioengineering. 55, 11835. 
Yoo, H., Antoniewicz, M. R., Stephanopoulos, G., Kelleher, J. K., 2008. Quantifying reductive carboxylation flux of glutamine to lipid in a brown adipocyte cell line. The Journal of biological chemistry. 283, 20621-7.

You, L., Berla, B., He, L., Pakrasi, H. B., Tang, Y. J., 2014. 13C-MFA delineates the photomixotrophic metabolism of Synechocystis sp. PCC 6803 under light- and carbon-sufficient conditions. Biotechnology journal. 9, 684-92.

Young, J. D., Shastri, A. A., Stephanopoulos, G., Morgan, J. A., 2011. Mapping photoautotrophic metabolism with isotopically nonstationary (13)C flux analysis. Metabolic engineering. 13, 65665.

Young, J. D., Walther, J. L., Antoniewicz, M. R., Yoo, H., Stephanopoulos, G., 2008. An elementary metabolite unit (EMU) based method of isotopically nonstationary flux analysis. Biotechnology and bioengineering. 99, 686-99.

Zhao, J., Shimizu, K., 2003. Metabolic flux analysis of Escherichia coli K12 grown on 13C-labeled acetate and glucose using GC-MS and powerful flux calculation method. Journal of biotechnology. 101, 101-17.

Zomorrodi, A. R., Suthers, P. F., Ranganathan, S., Maranas, C. D., 2012. Mathematical optimization applications in metabolic networks. Metabolic engineering. 14, 672-86.

Zupke, C., Stephanopoulos, G., 1994. Modeling of Isotope Distributions and Intracellular Fluxes in Metabolic Networks Using Atom Mapping Matrixes. Biotechnology Progress. 10, 489-498. 


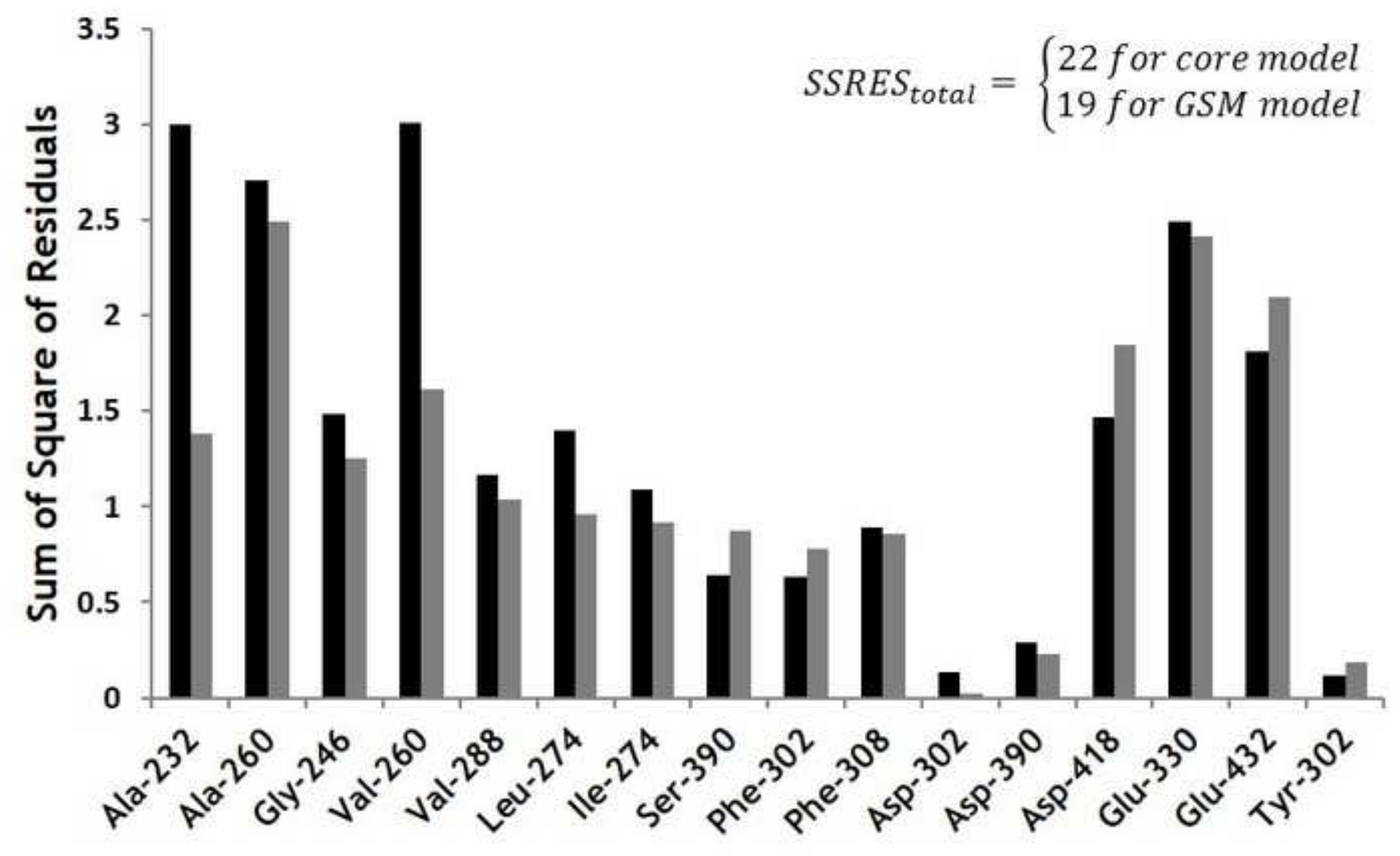


Figure $2 a$

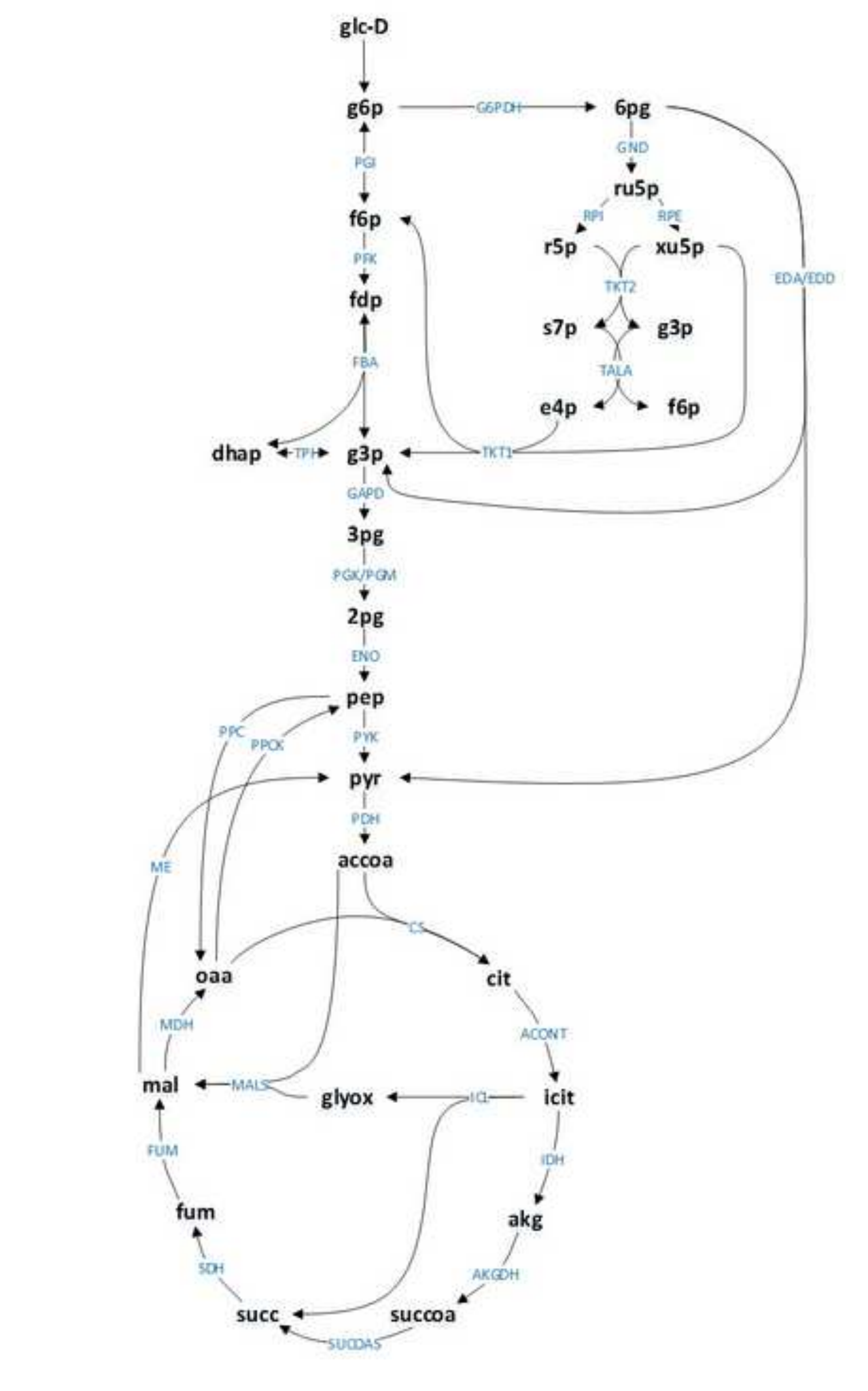

.

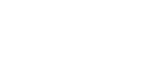

-

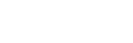

-

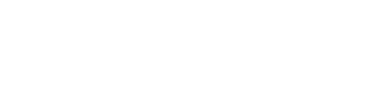

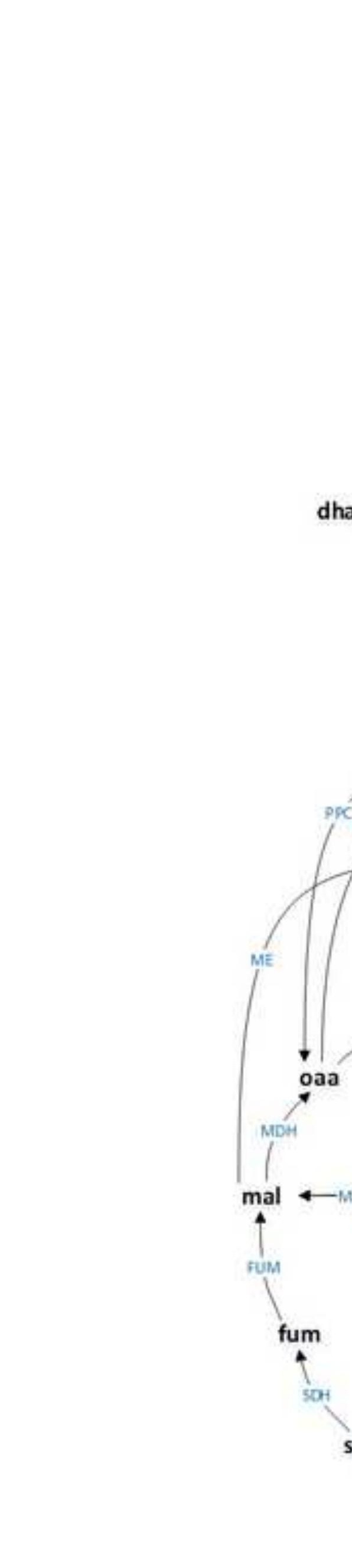



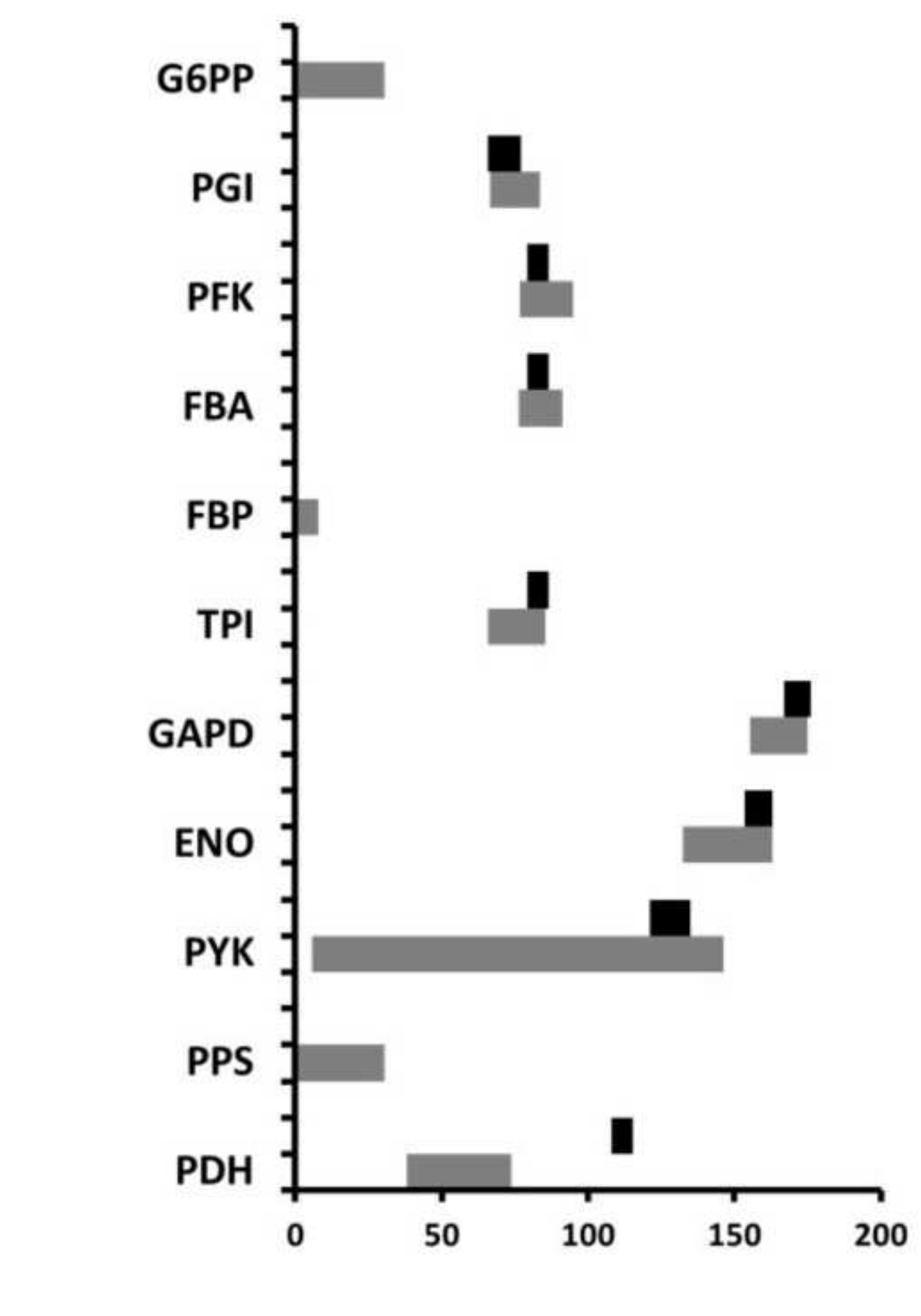

.
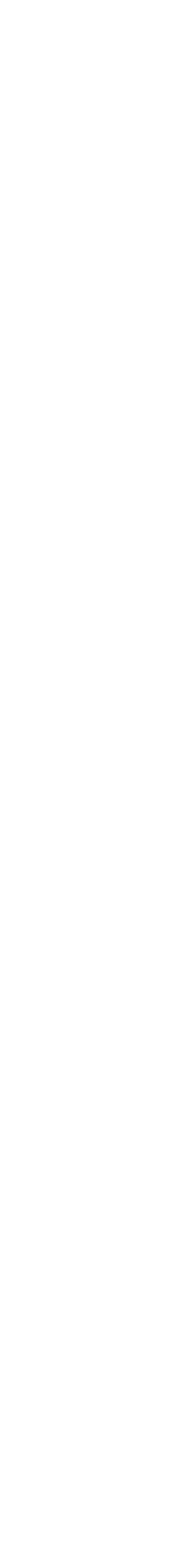


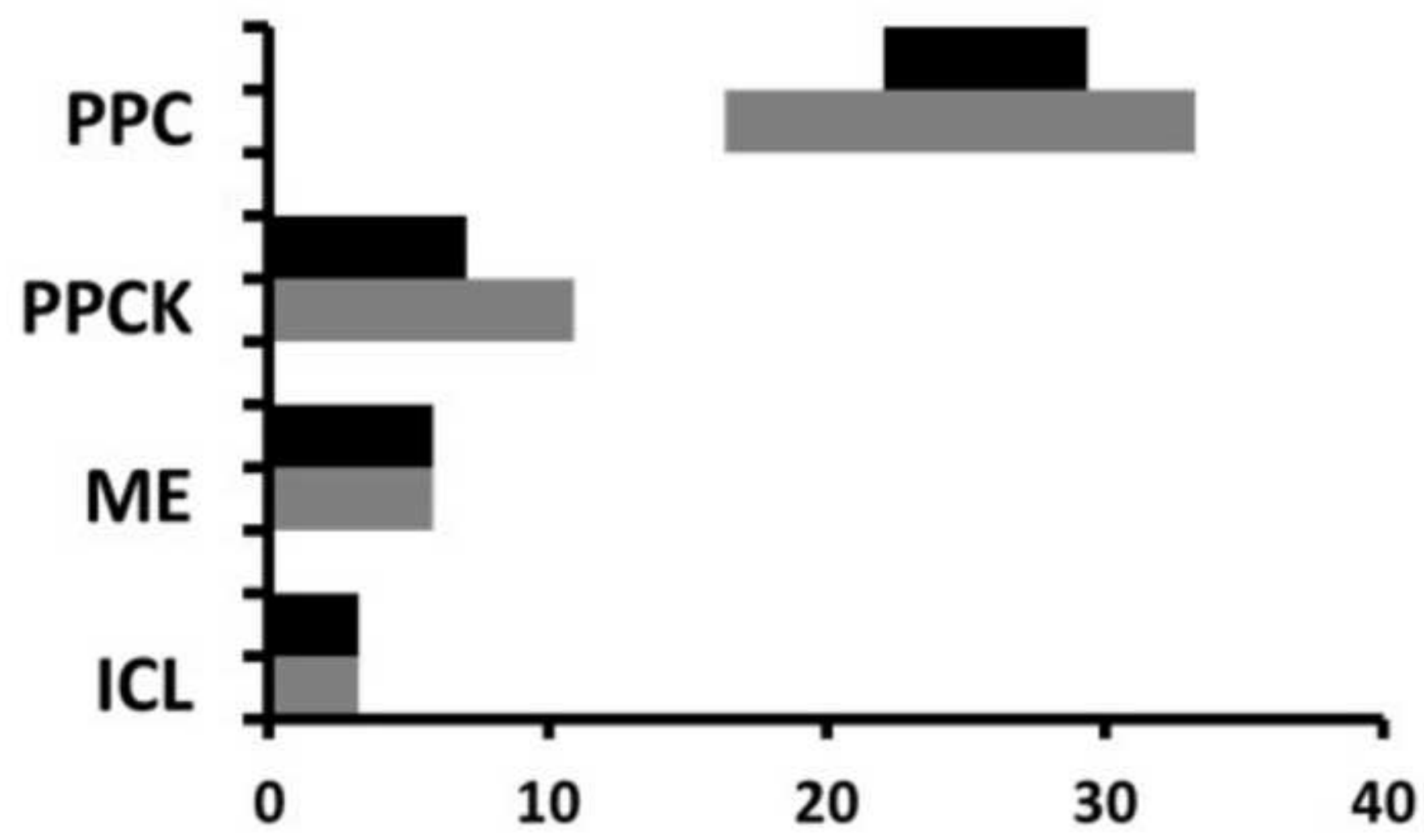

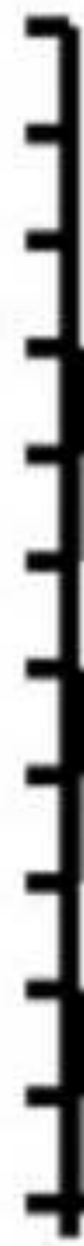

0

10

20

30

40 


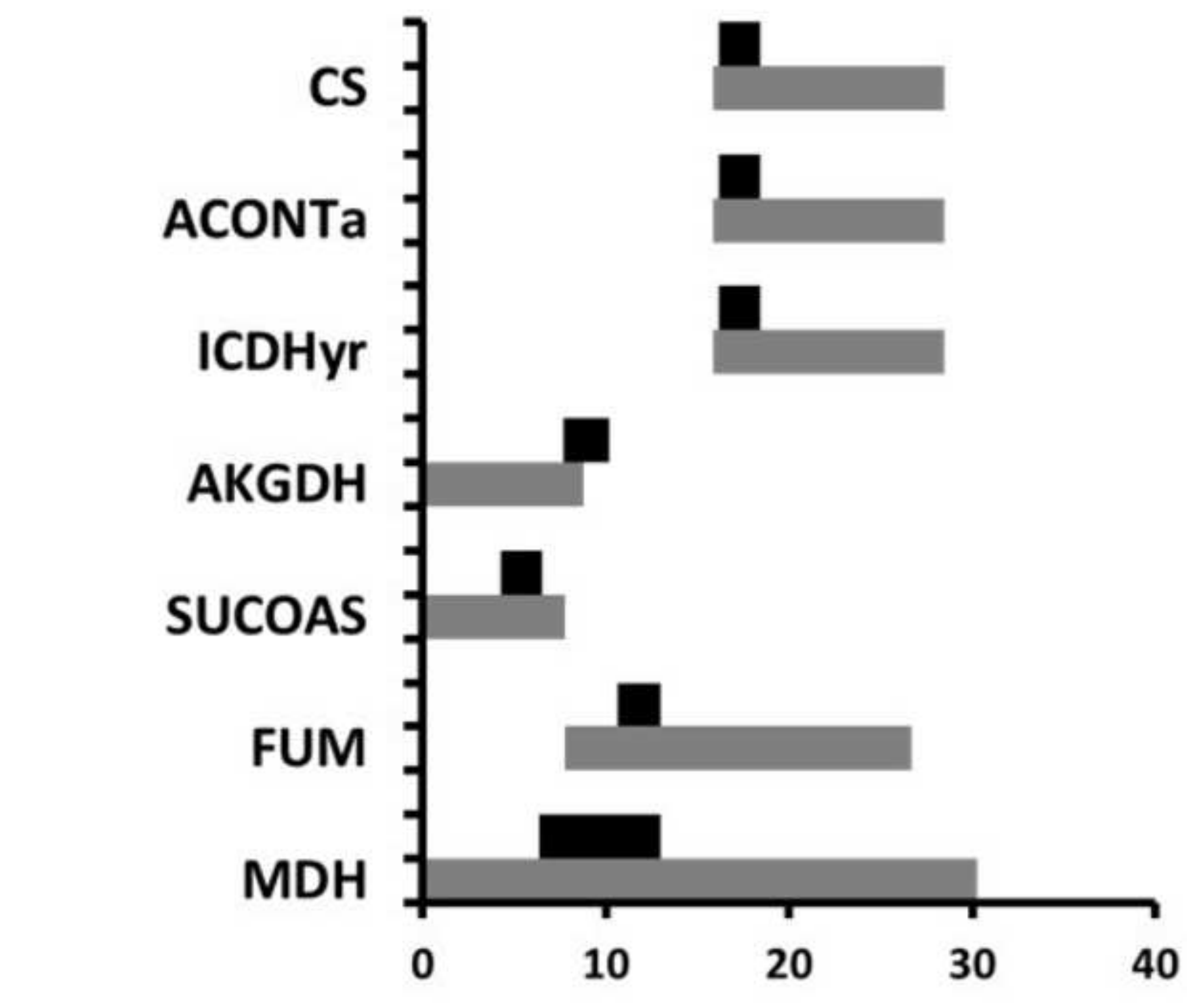




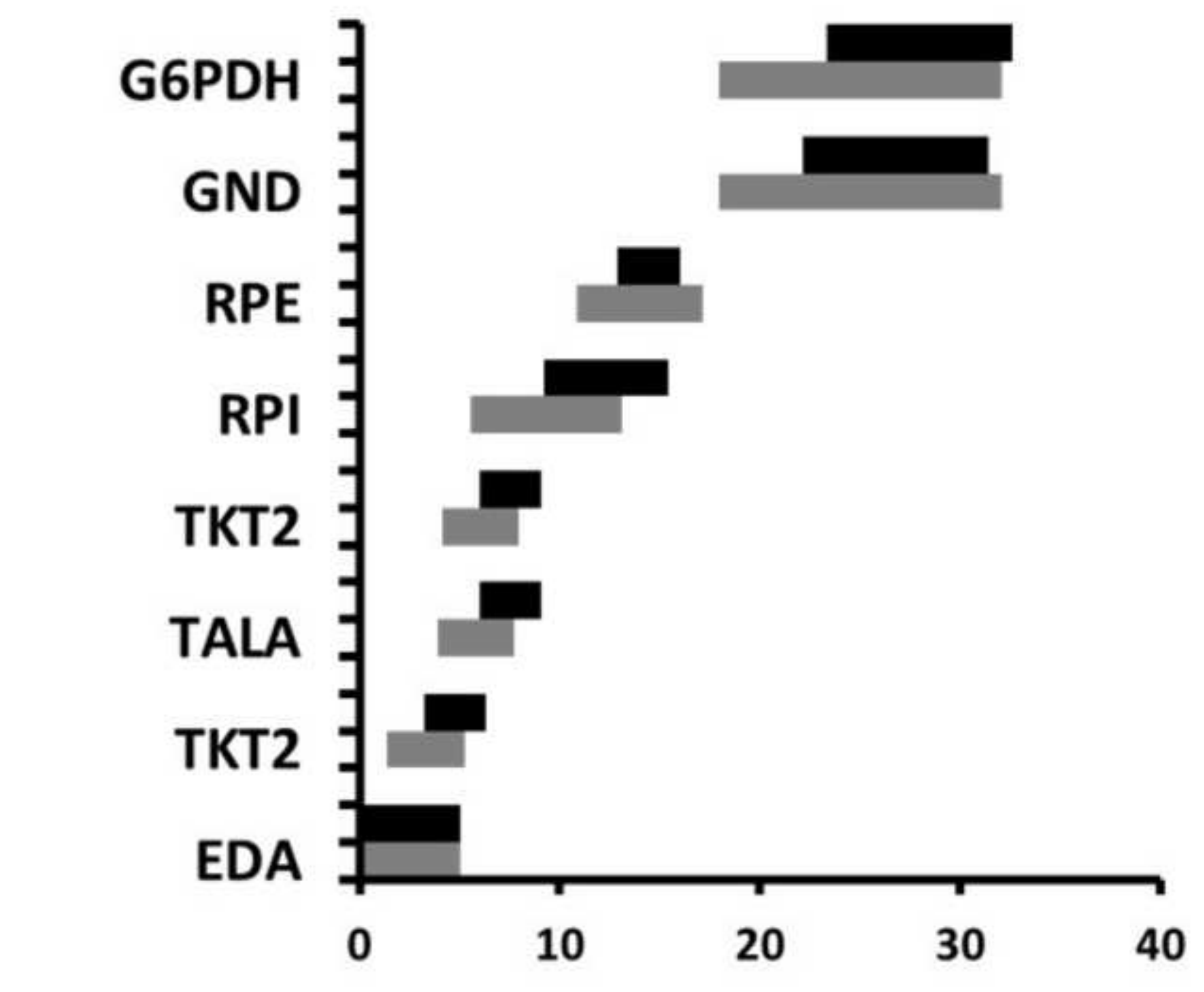


NADDH
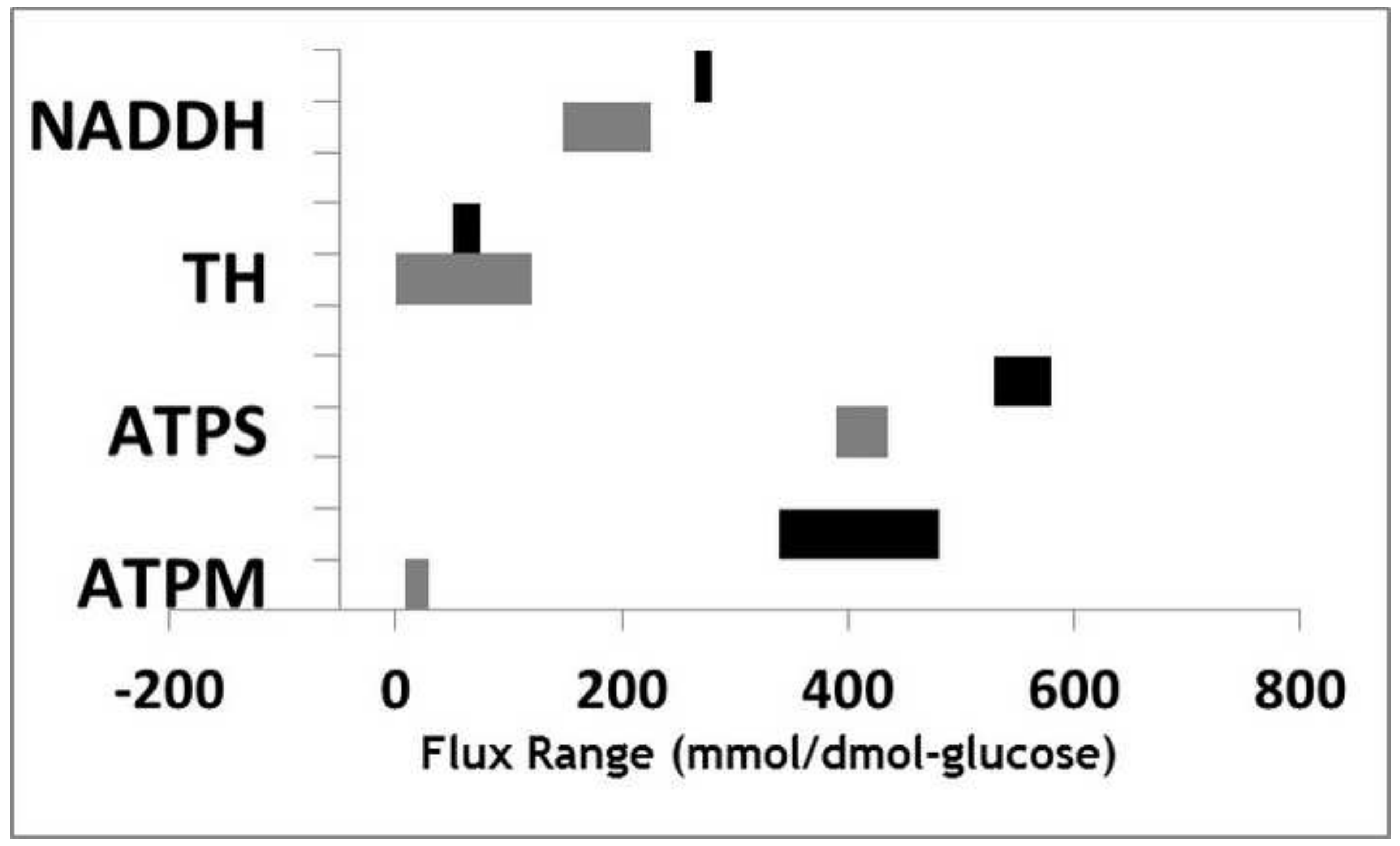

Flux Range (mmol/dmol-glucose)

$-200$

0

200

800 


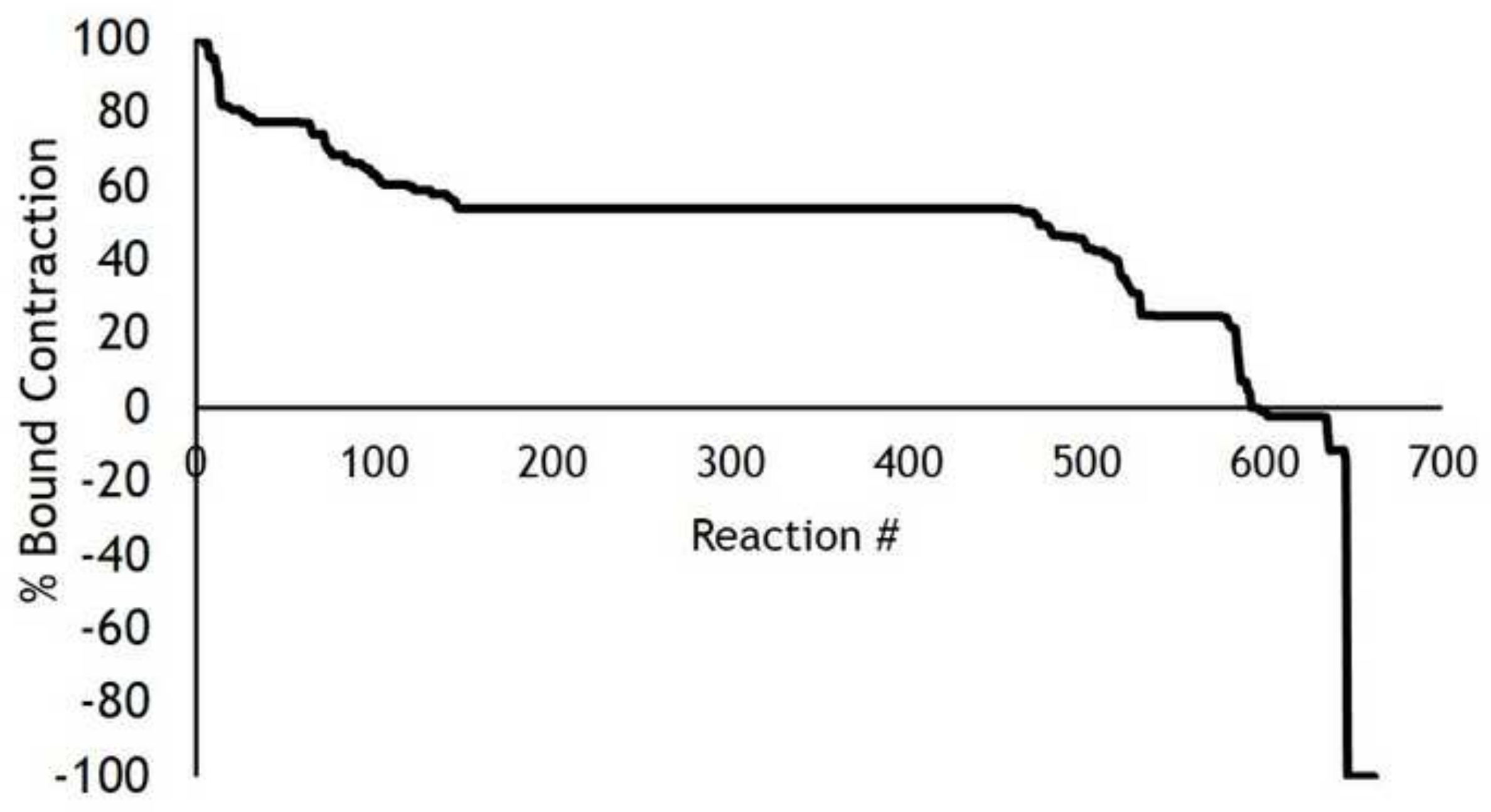




\section{Tables}

Table 1: $\chi^{2}$ degrees of freedom for the core model and the genome-scale model. Statistical significance requires that the number of degrees of freedom be positive as a $\chi^{2}$ value is defined only for positive integers. The difference in the number of degrees of freedom estimated using free fluxes and based on the EMU network points to an inherent flaw in using the free fluxes as the number of model parameters.

\begin{tabular}{|l|ll|}
\hline & Degrees of Freedom & Maximum SSRES \\
\hline Core model & 55 & 96 \\
$\begin{array}{l}\text { Genome-scale model } \\
\text { (based on free fluxes) }\end{array}$ & -30 & Not defined \\
$\begin{array}{l}\text { Genome-scale model } \\
\text { (based on EMU network) }\end{array}$ & 27 & 44 \\
\hline
\end{tabular}


Table 2: Additional suggested MS measurements for resolving various alternate routes.

\begin{tabular}{|cccc|}
\hline $\begin{array}{c}\text { Central } \\
\text { Metabolic } \\
\text { pathway }\end{array}$ & $\begin{array}{c}\text { Alternate } \\
\text { Pathway }\end{array}$ & $\begin{array}{c}\text { Metabolite } \\
\text { measurement } \\
\text { candidate }\end{array}$ & $\begin{array}{c}\text { Measurement } \\
\text { Type }\end{array}$ \\
\hline Lower glycolysis & $\begin{array}{c}\text { Methylglyoxal } \\
\text { pathway }\end{array}$ & methylglyoxal & $\begin{array}{c}\text { Time-course MS } \\
\text { with known pool } \\
\text { size }\end{array}$ \\
TCA cycle & Arginine & $\gamma$-aminobutyrate & $\begin{array}{c}\text { Time-course MS } \\
\text { with known pool } \\
\text { size }\end{array}$ \\
degradation & G6PP & Intracellular & glucose \\
cycle & & Steady-state MS \\
\hline
\end{tabular}

